\title{
Stable and high power 515-nm lasers for the space gravitational wave detector: $\mathrm{DECIGO}$
}

\author{
Aru Suemasa, Shunsuke Ohtsuka, Ayumi Shimo-oku, \\ Masaki Nakamori, Mitsuru Musha
}

Institute for Laser Science, University of Electro-communications 


\section{Outline}

- Gravitational wave (GW) \& space GW detector

- Frequency stabilization

- Balanced detection

- Intensity stabilization at $200 \mathrm{kHz}$

- Cascaded amplifier for B-DECIGO

- Summary \& future prospects 


\section{Gravitational Wave}

GW sources

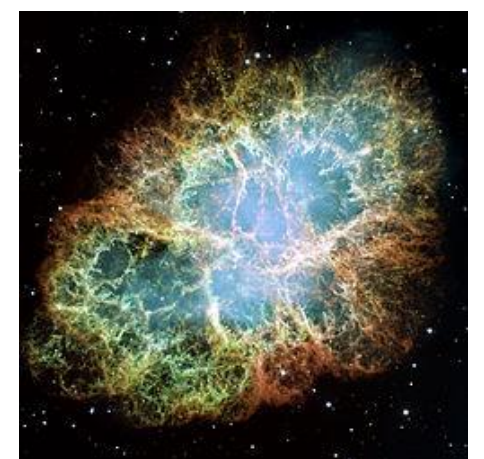

NASA, ESA, J. Hester and A. Loll (Arizona State University)

Explosion of supernovae
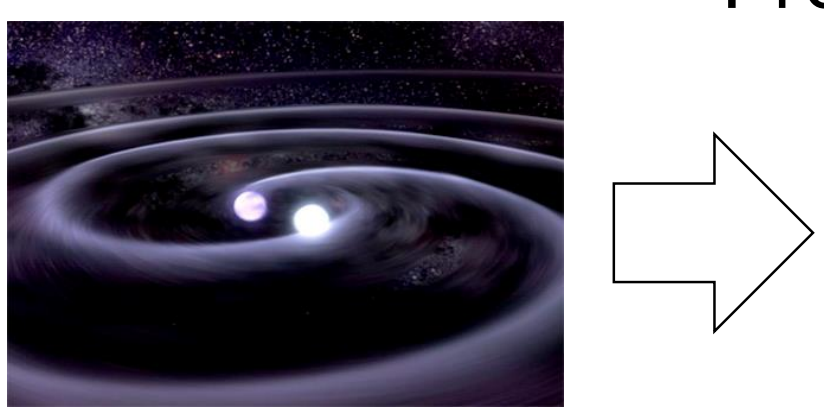

NASA/Tod Strohmayer (GSFC)/Dana Berry (Chandra X-Ray Observatory)

NS binary

$\Rightarrow$ Spatial distortion(temporal variation )
Propagates as a transvers wave

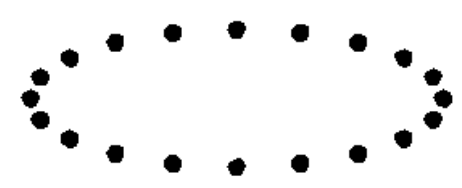
in General Theory of Relativity

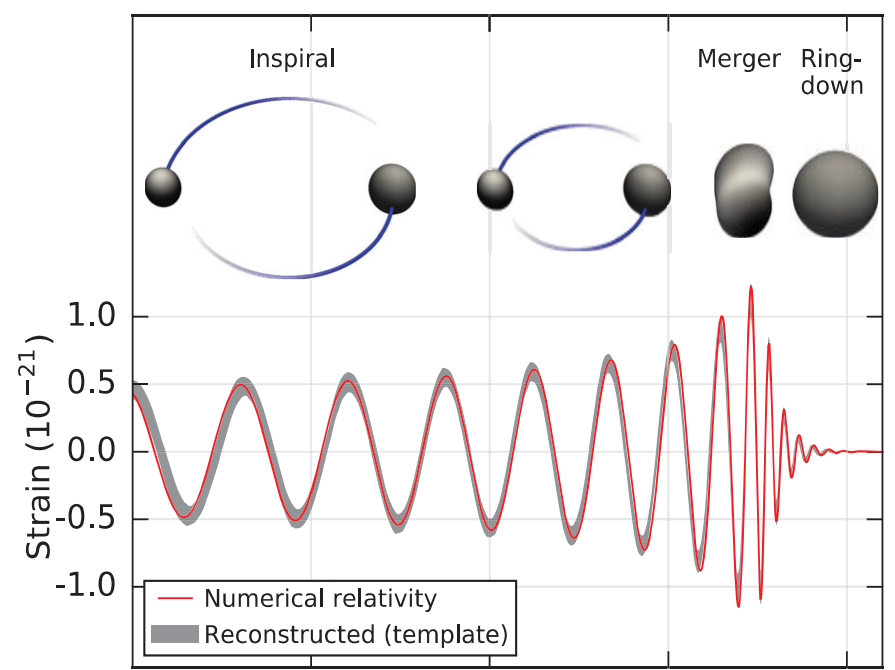

B.P. PHYSICAL REVIEW LETTERS 116, 061102 (2016)

The first direct detection of GW was achieved by adv.LIGO team

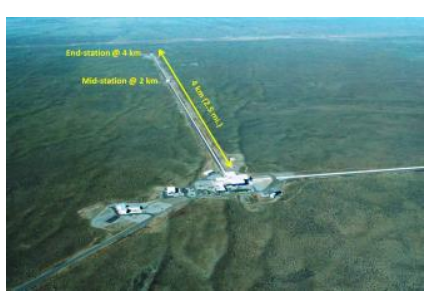

Ligo.caltech.edu HP long-baseline Michelson interferometer

$$
\Delta \mathrm{L} / \mathrm{L}<10^{-22}
$$

ICSO20189/10/2018@Crete 


\section{Space gravitational wave detector}

Space GW detector DECIGO/B-DECIGO
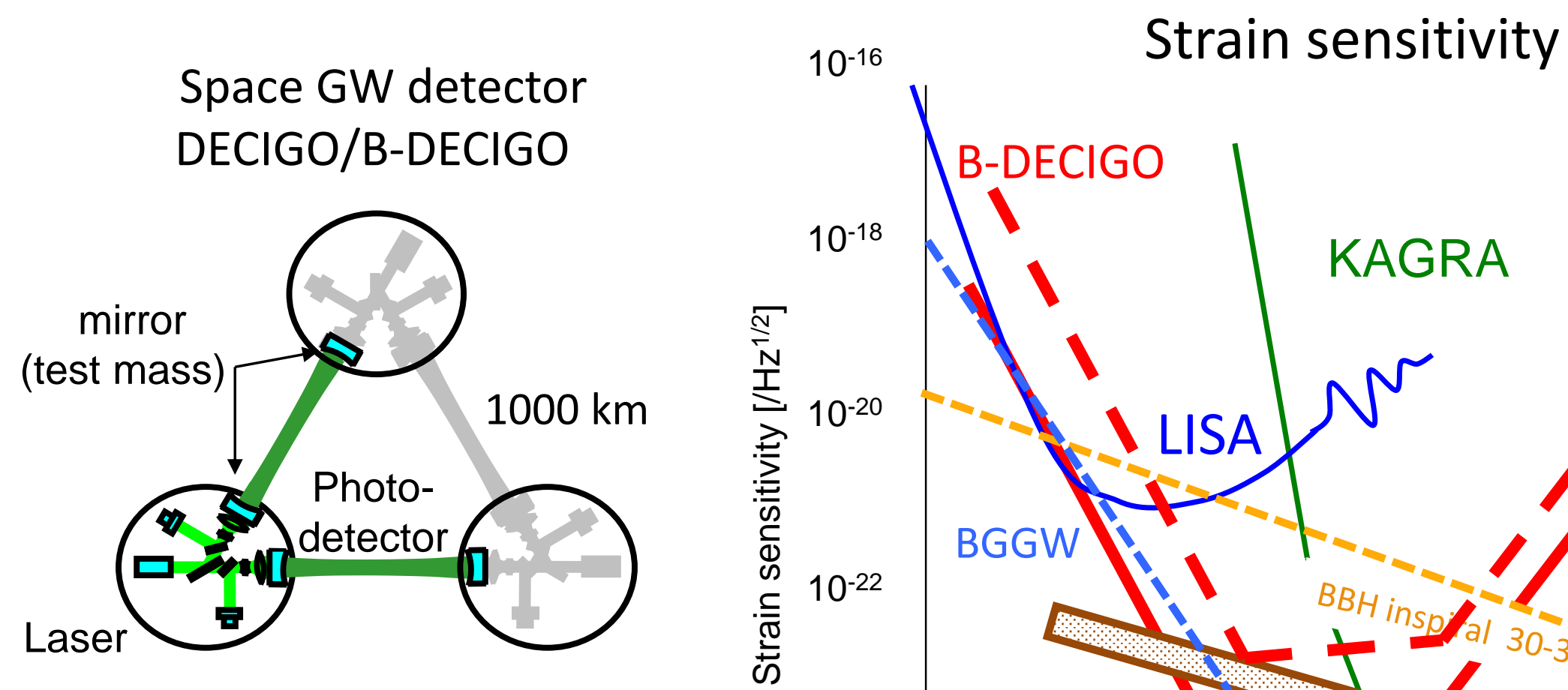

- Strain sensitivity : $\Delta L / L=10^{-24 / V} \mathrm{~Hz}^{10^{-24}}$

- Observation band :0.1 10 Hz

- Planed to be launched in 2035

- Milestone mission B-DEIGO

- Target: IMBH binary, NS binary, BGGW

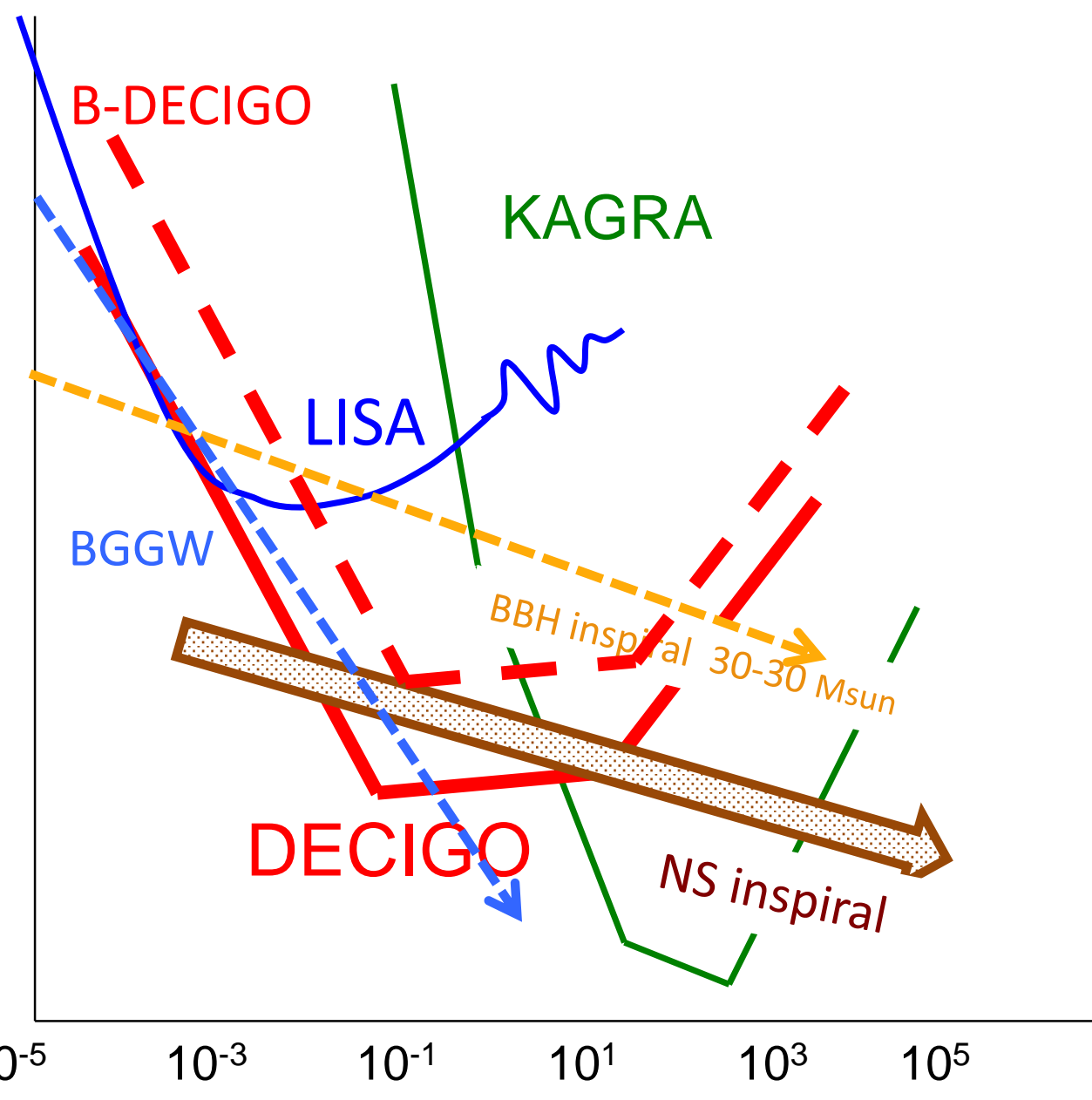

frequency $[\mathrm{Hz}]$

ICSO2018 9/10/2018@Crete 


\section{Light source for DECIGO/B-DECIGO}

Requirement values for light source

\begin{tabular}{|c|c|c|c|c|}
\hline & $\begin{array}{l}\text { Wave } \\
\text { length } \\
\mu \mathrm{m}\end{array}$ & $\begin{array}{l}\text { Frequency noise } \\
\mathrm{Hz} / \mathrm{VHz} @ 1 \mathrm{~Hz}\end{array}$ & $\begin{array}{c}\text { Relative intensity noise } \\
1 / \mathrm{vHz} @ 1 \mathrm{~Hz} \text { and } 200 \mathrm{kHz}\end{array}$ & $\begin{array}{l}\text { Output } \\
\text { power } \\
\text { W }\end{array}$ \\
\hline B-DECIGO & 0.5 & \multirow{2}{*}{$\frac{1}{1} d f / f=1.6 \times 10^{-15}$} & $1 \times 10^{-8}$ & 2 \\
\hline DECIGO & 0.5 & & $1 \times 10^{-8}$ & 10 \\
\hline
\end{tabular}

Developments of Breadboard models (BBM1, BBM2)

$\mathrm{I}_{2}$-stabilized Yb:fiber DFB laser (515 nm)

(high long-term stability, robustness)

$+$

Intensity stabilization@1 Hz and 200 kHz

$+$

High power (10 W) @ 515 nm

ICS020189/10/2018@Crete 


\section{Outline}

- Gravitational wave (GW) \& space GW detector

- Frequency stabilization

- Balanced detection

- Intensity stabilization at $200 \mathrm{kHz}$

- Cascaded amplifier for B-DECIGO

- Summary \& future prospects 


\section{Schematic diagram of Breadboard model(BBM)}

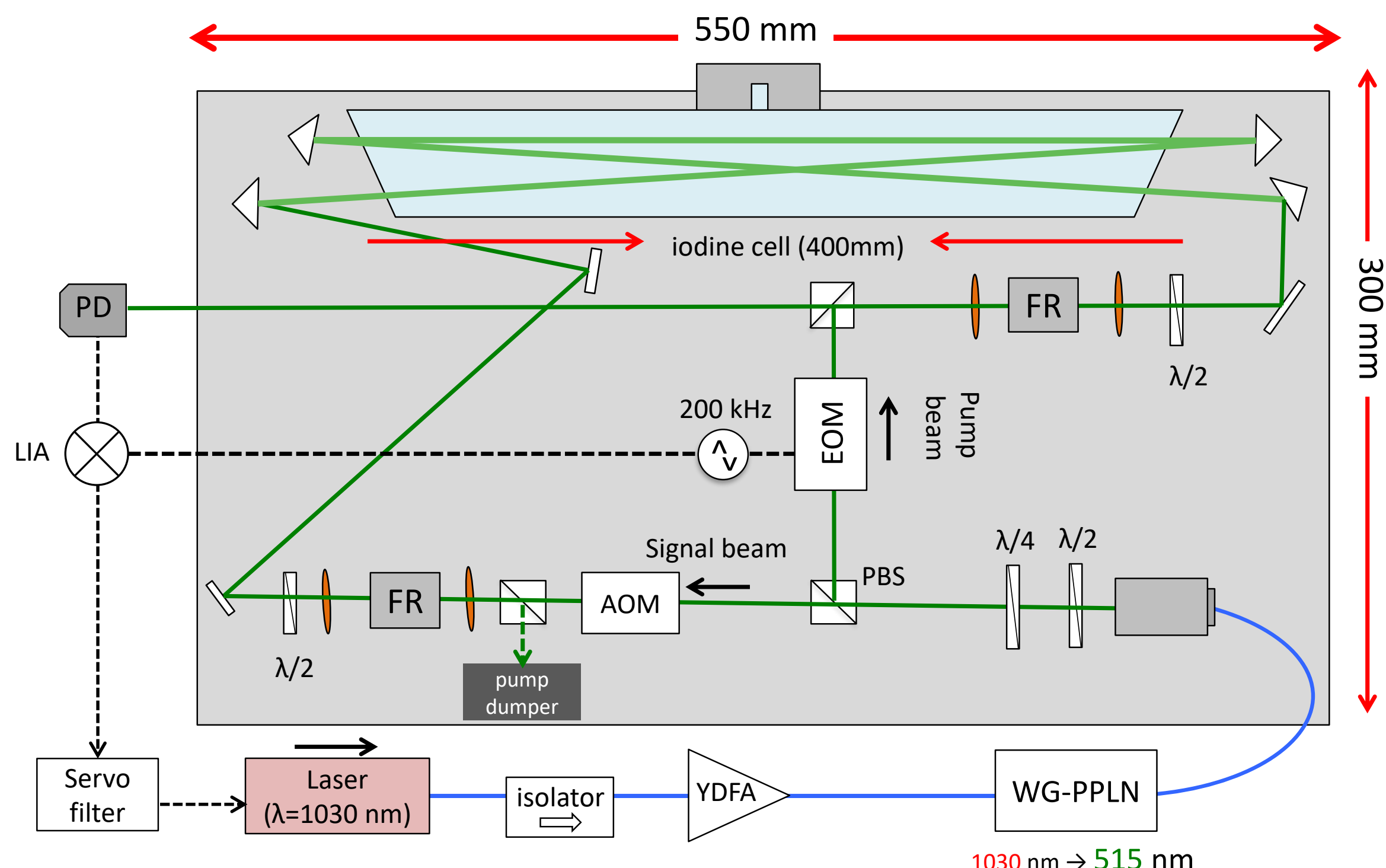

ICSO2018 9/10/2018@Crete 


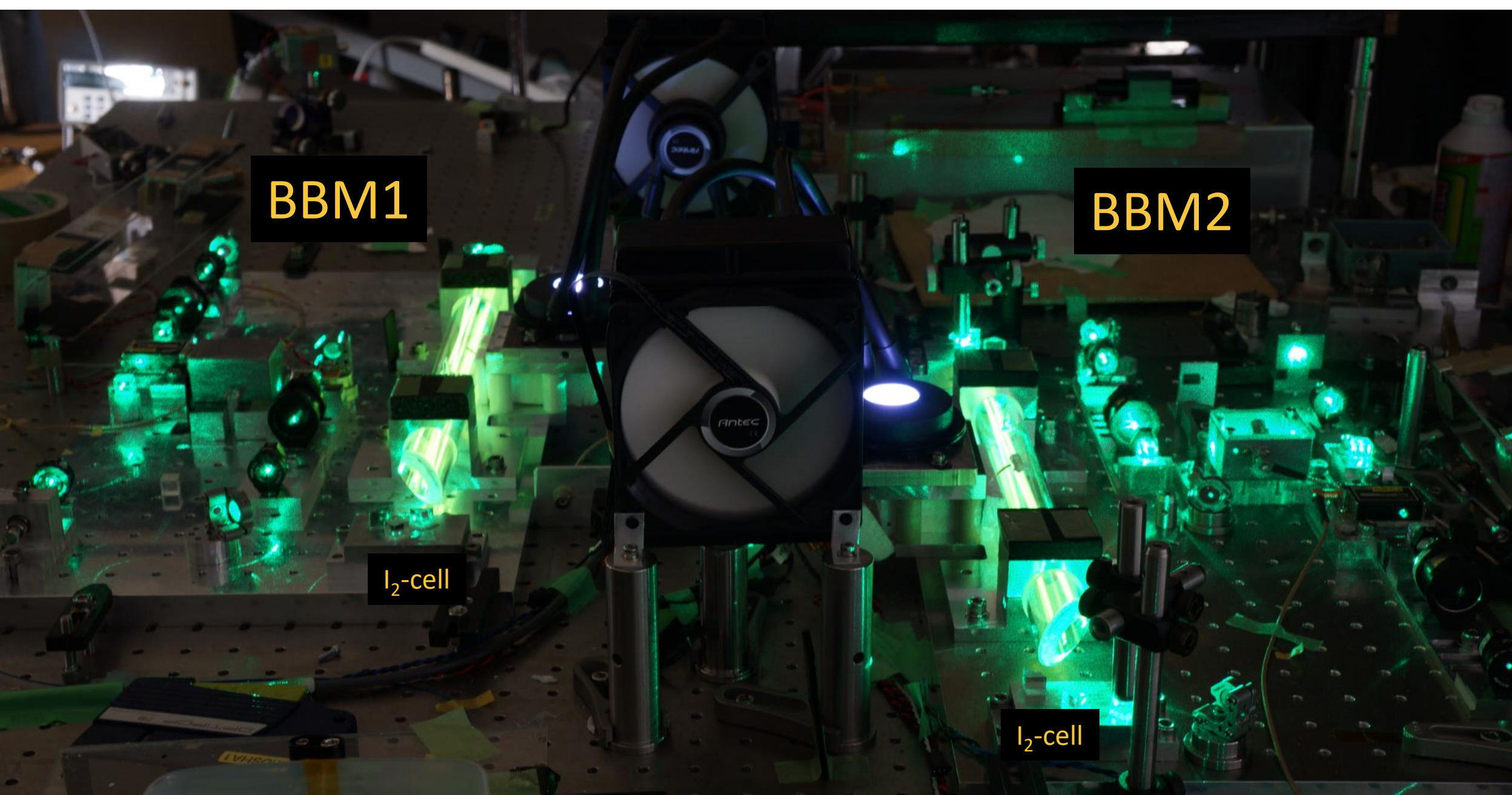

ICSO2018 9/10/2018@Crete 


\section{Frequency stability (error signal)}

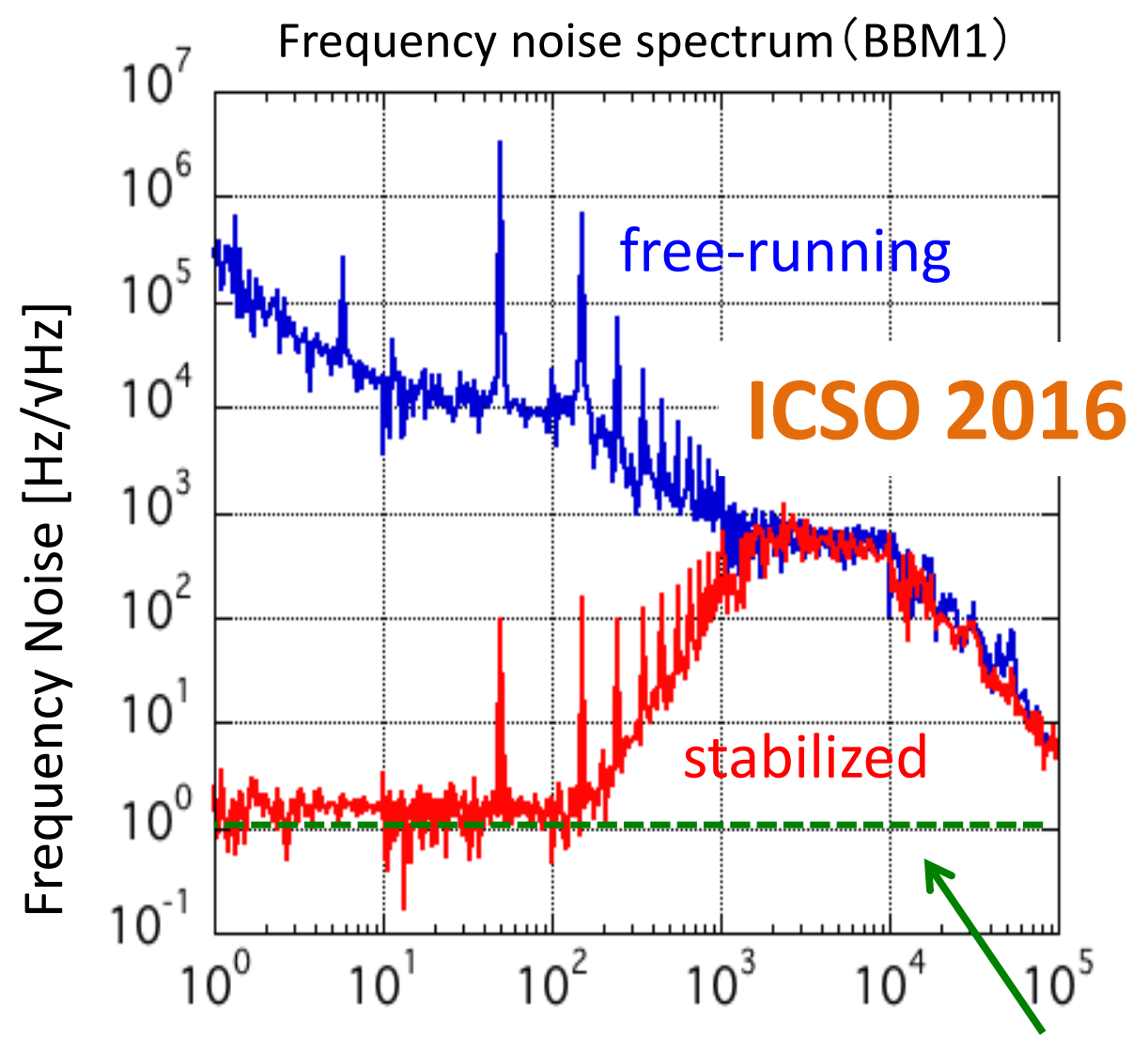

Frequency $[\mathrm{Hz}]$

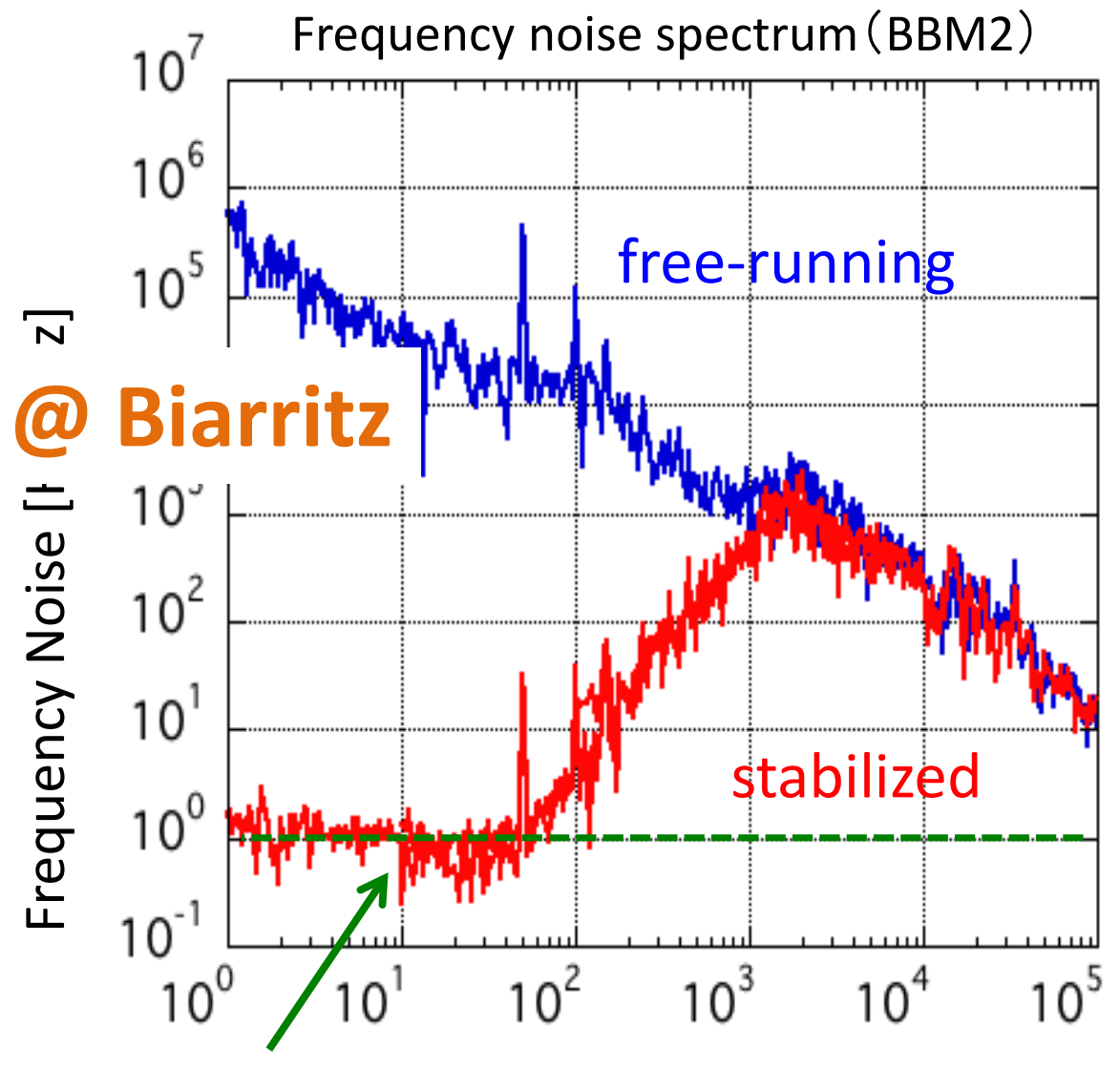

Frequency $[\mathrm{Hz}]$

of DECIGO/B-DECIGO

$\Delta f=1 \mathrm{~Hz} / \mathrm{VHz}$ (in-loop signal) @below $10 \mathrm{~Hz}$ Satisfies required value. 


\section{Frequency stability (out-of-loop)}
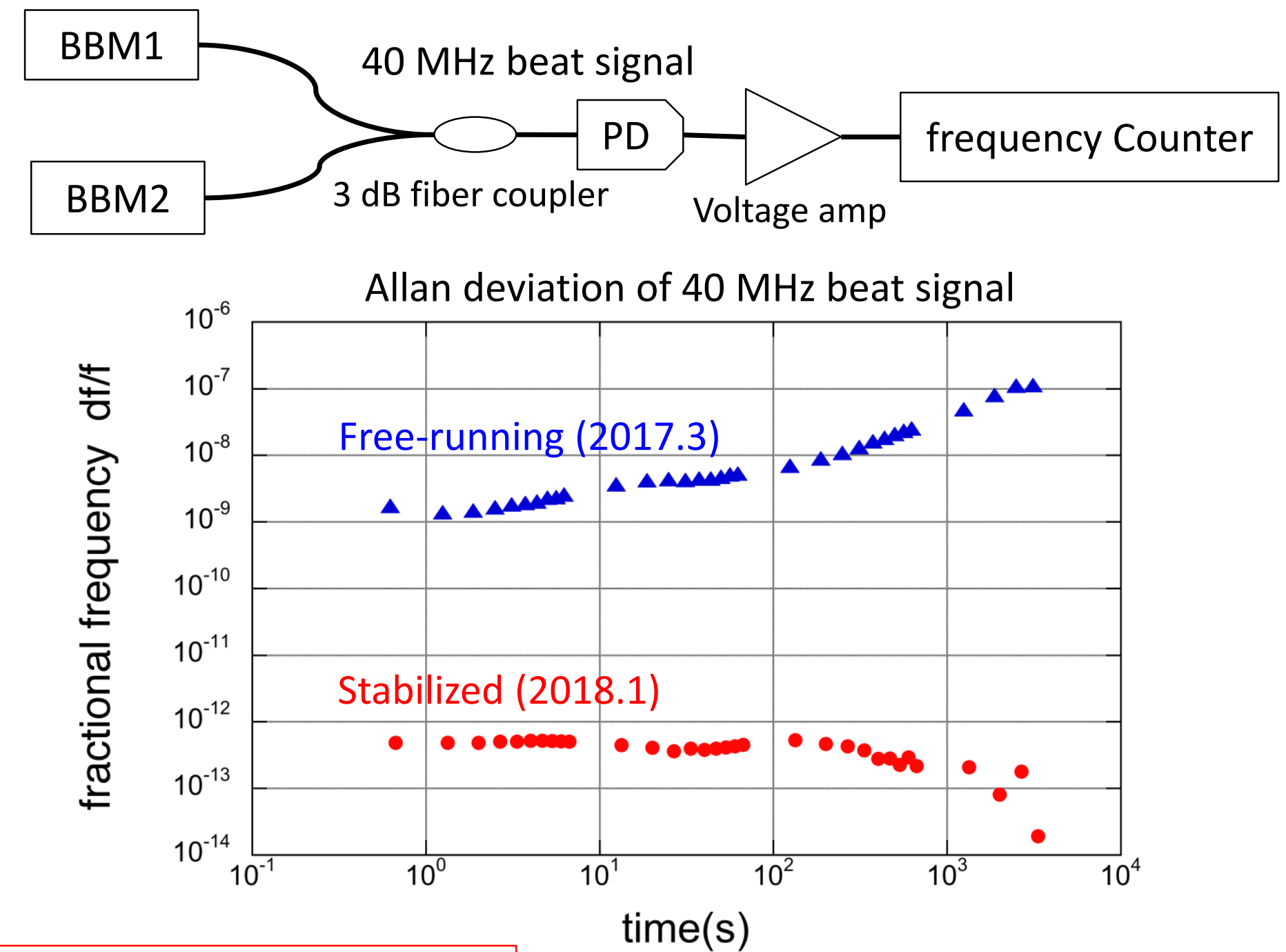

$$
d f / f=10^{-13} \text { level @1s }
$$$$
d f / f=10^{-14} \text { level @3000s }
$$ 


\section{Frequency stability (out-of-loop)}
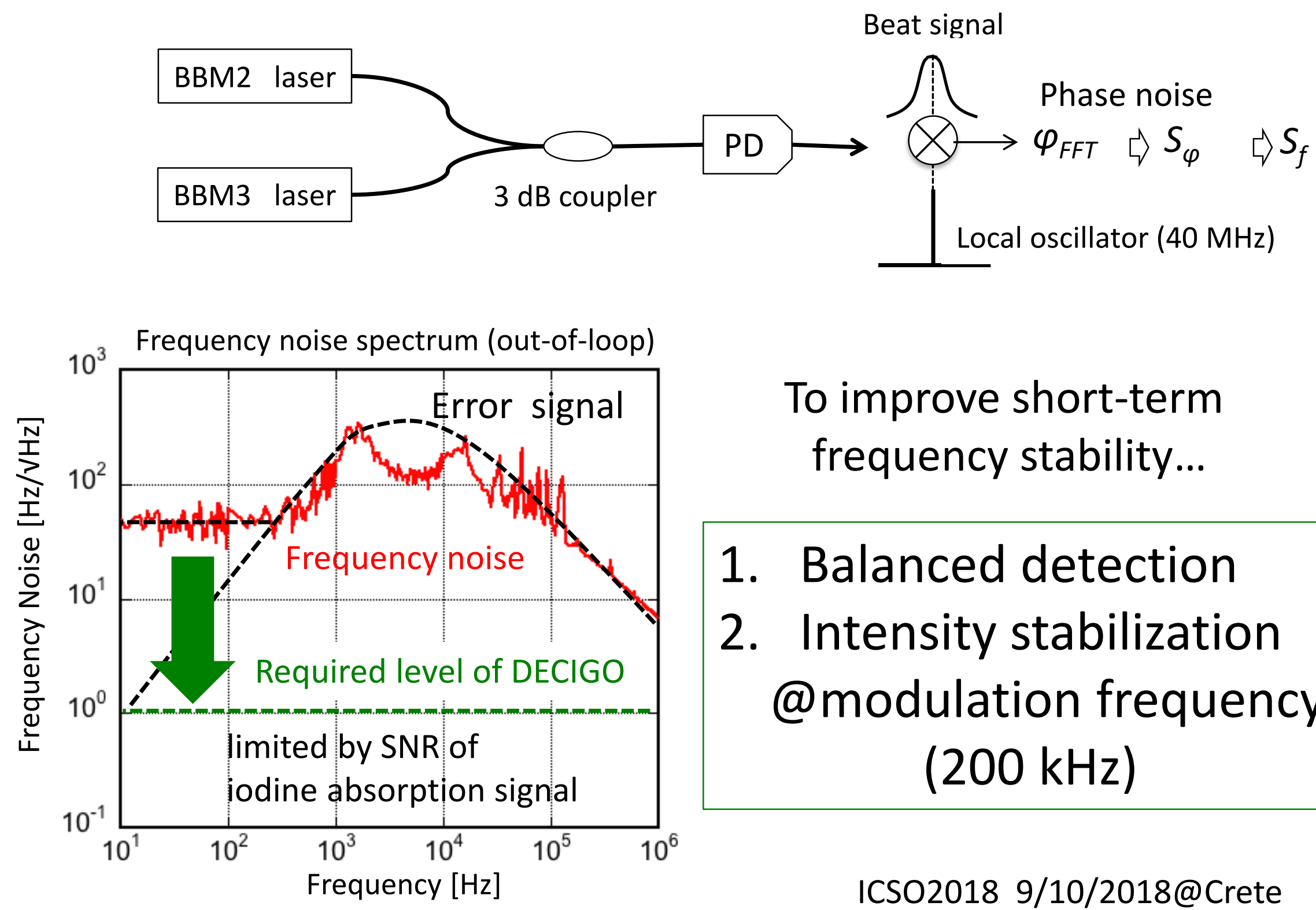

To improve short-term frequency stability...

1. Balanced detection

2. Intensity stabilization @modulation frequency (200 kHz) 


\section{RIN of amplified fiber DFB laser}

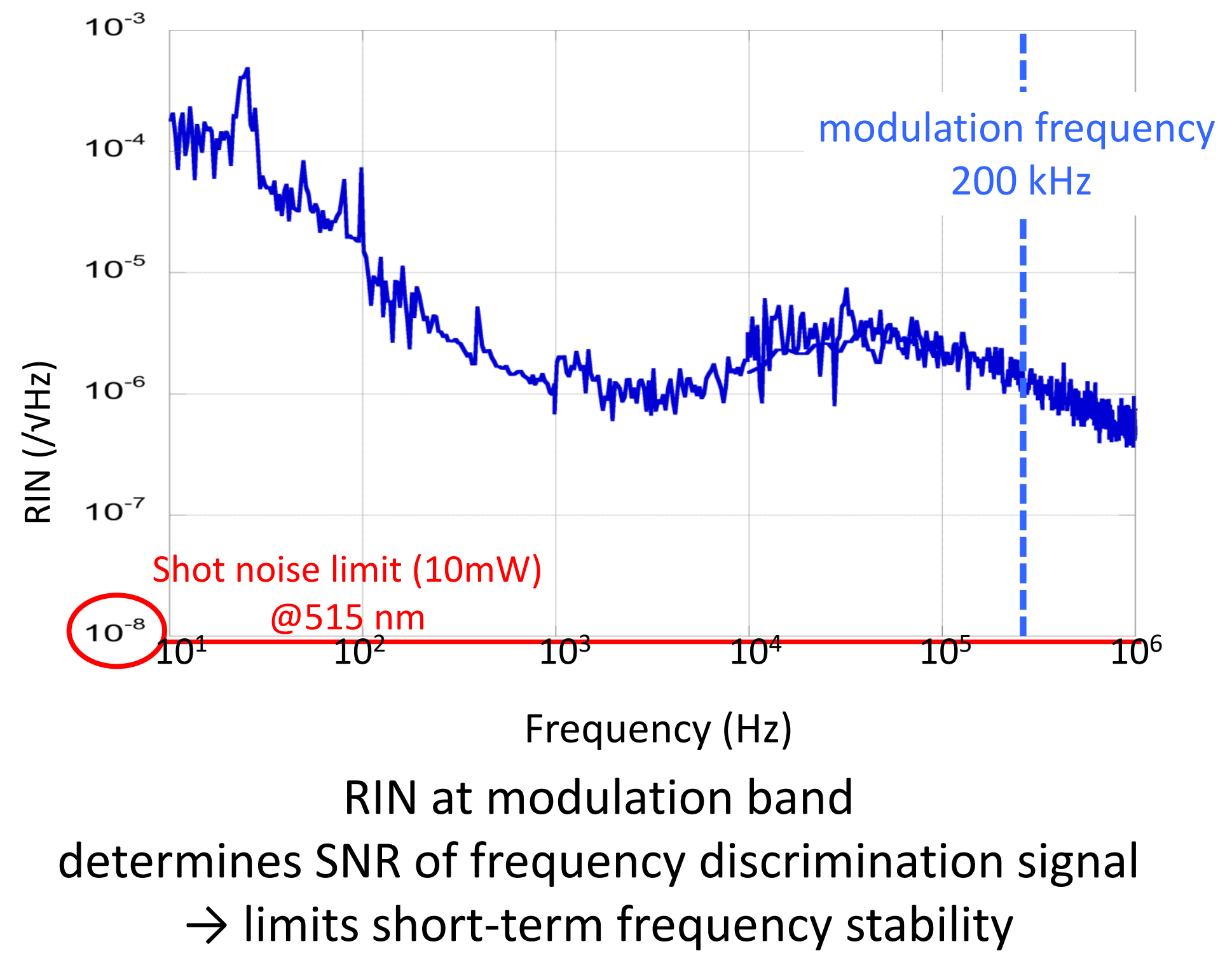




\section{Outline}

- Gravitational wave (GW) \& space GW detector

- Frequency stabilization

- Balanced detection

- Intensity stabilization at $200 \mathrm{kHz}$

- Cascaded amplifier for B-DECIGO

- Summary \& future prospects 


\section{Balanced detection(1)}

Auto-balanced differential photo detector is installed in BBM to eliminate the common mode noise from laser intensity noise

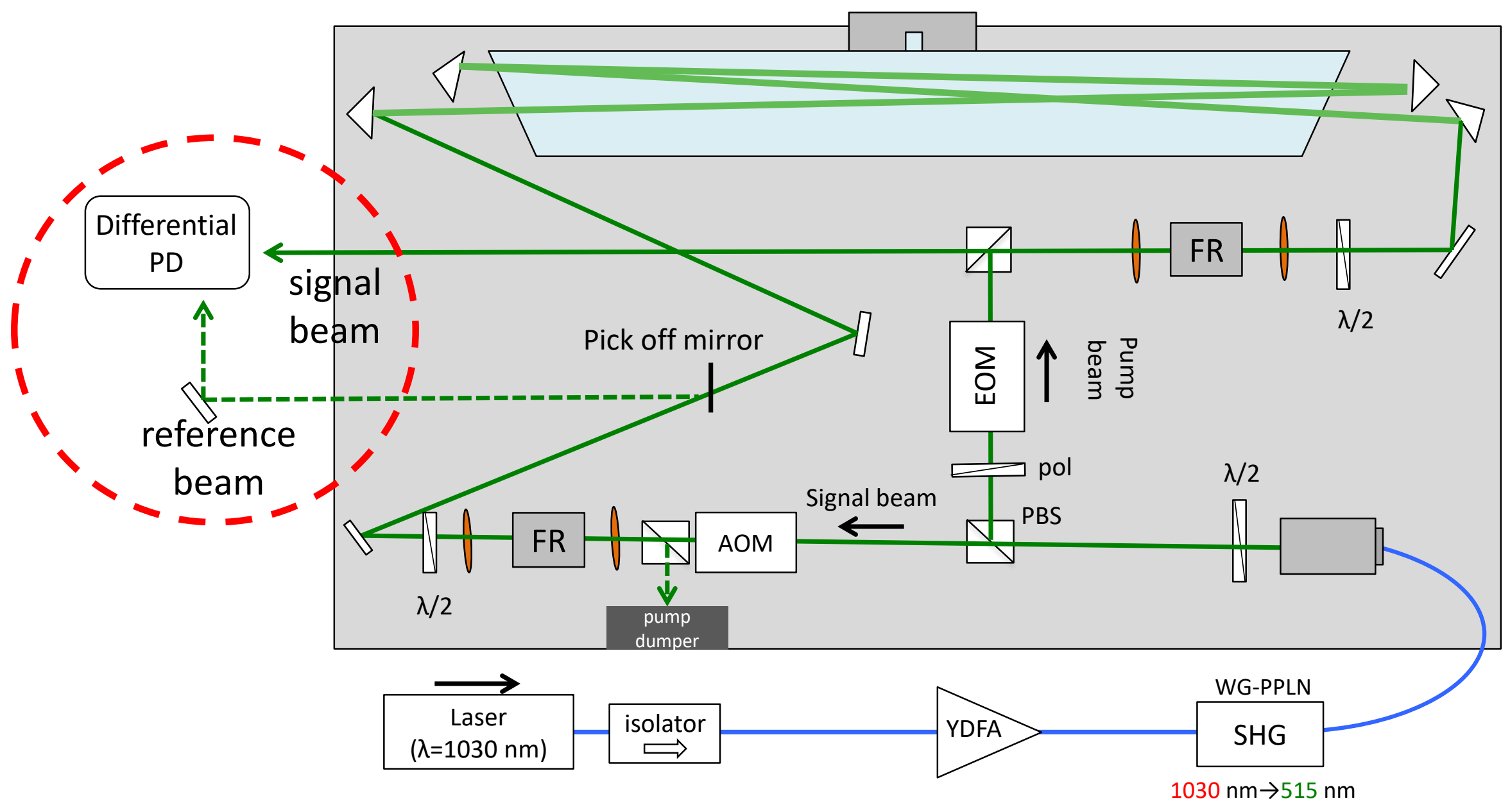




\section{Balanced detection (2)}
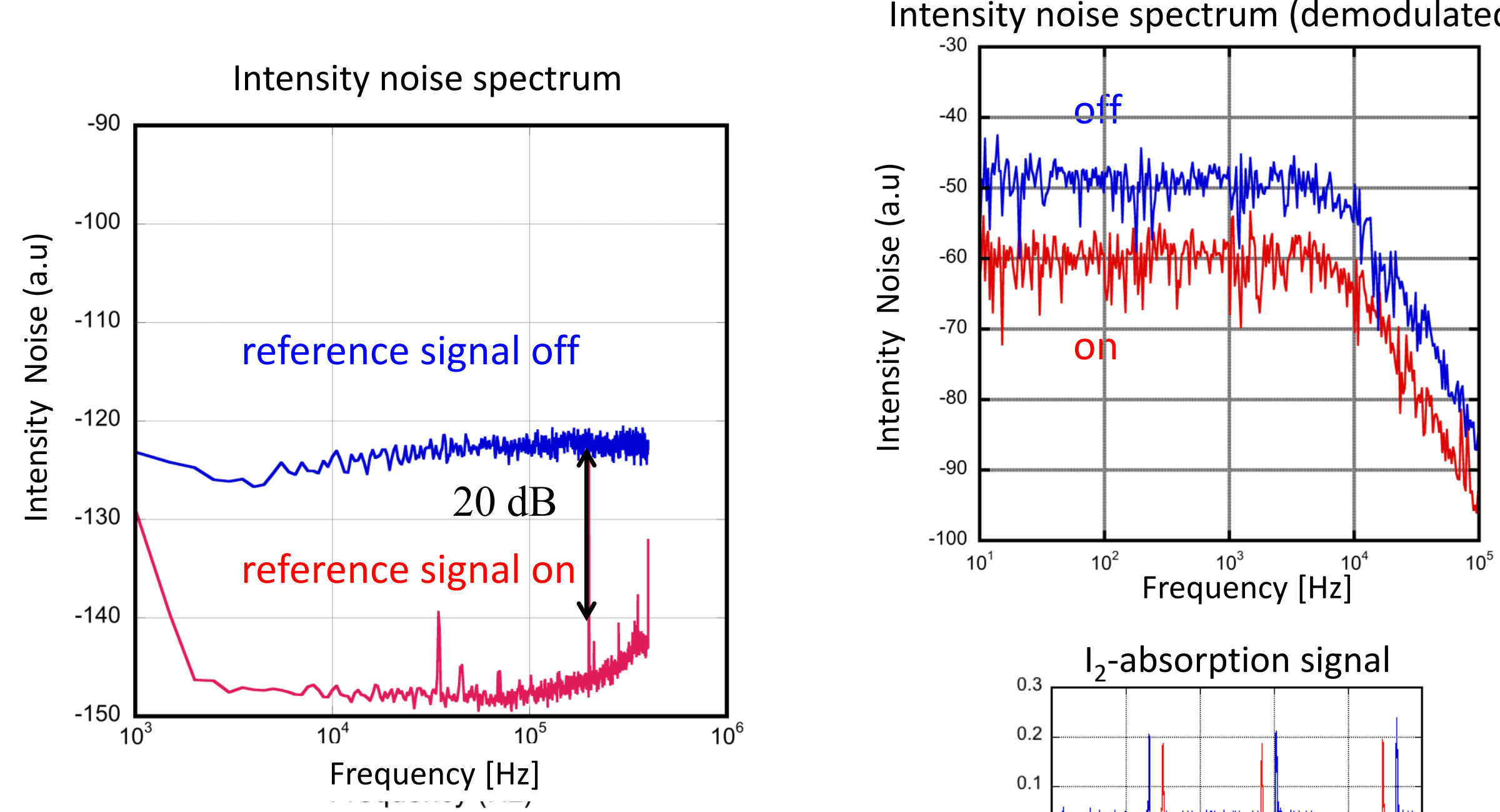

Balanced detection improves short-term frequency stability!

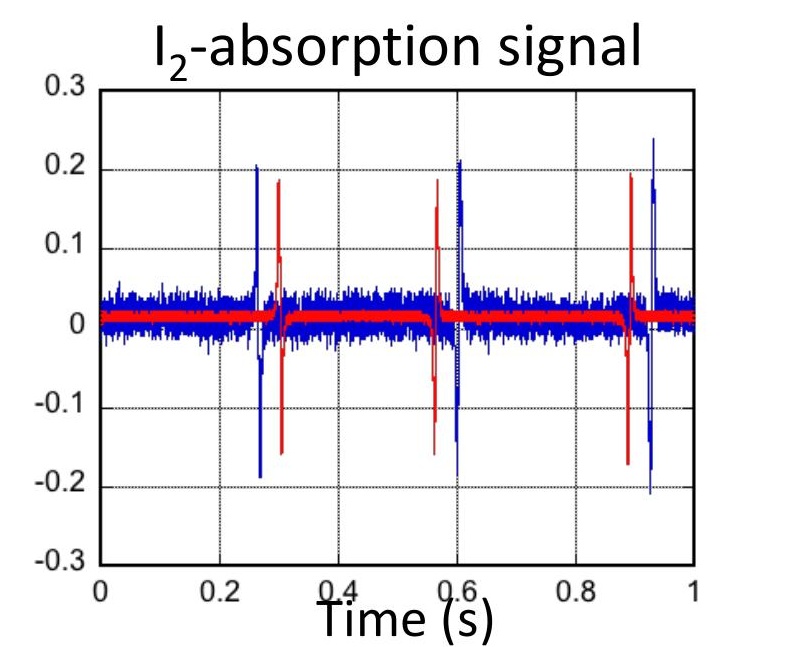




\section{Outline}

- Gravitational wave (GW) \& space GW detector

- Frequency stabilization

- Balanced detection

- Intensity stabilization at $200 \mathrm{kHz}$

- Cascaded amplifier for B-DECIGO

- Summary \& future prospects 


\section{Active intensity stabilization@200 kHz}

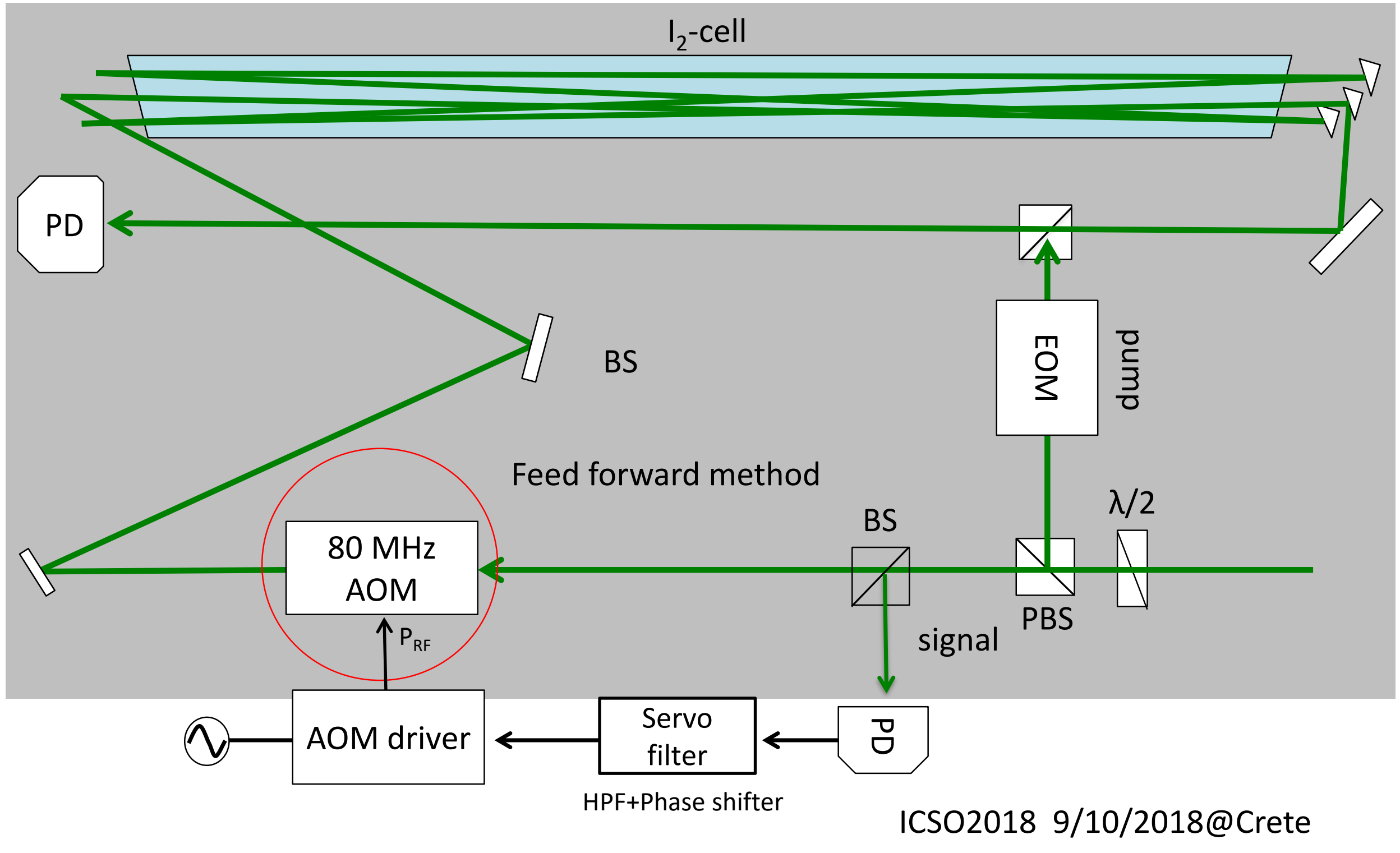




\section{Intensity stabilization at $200 \mathrm{kHz}$}

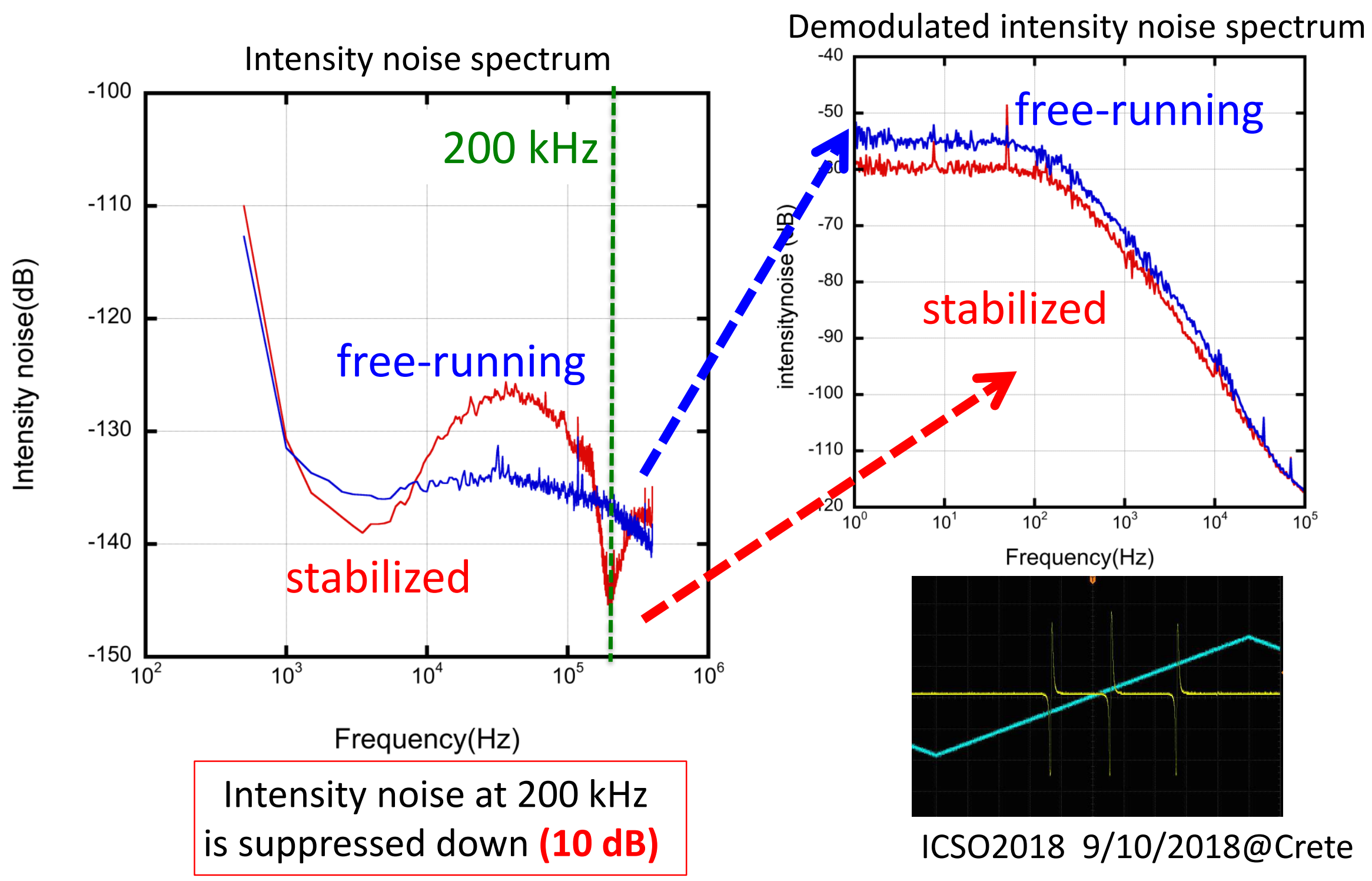




\section{Improvement of short-term stability}

\section{Beat note}

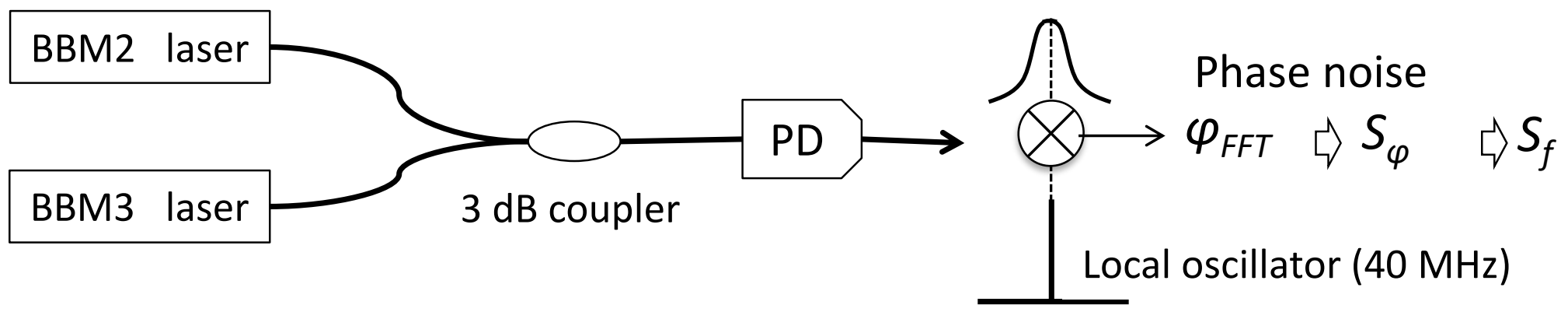

frequency noise spectrum (out-of-loop)

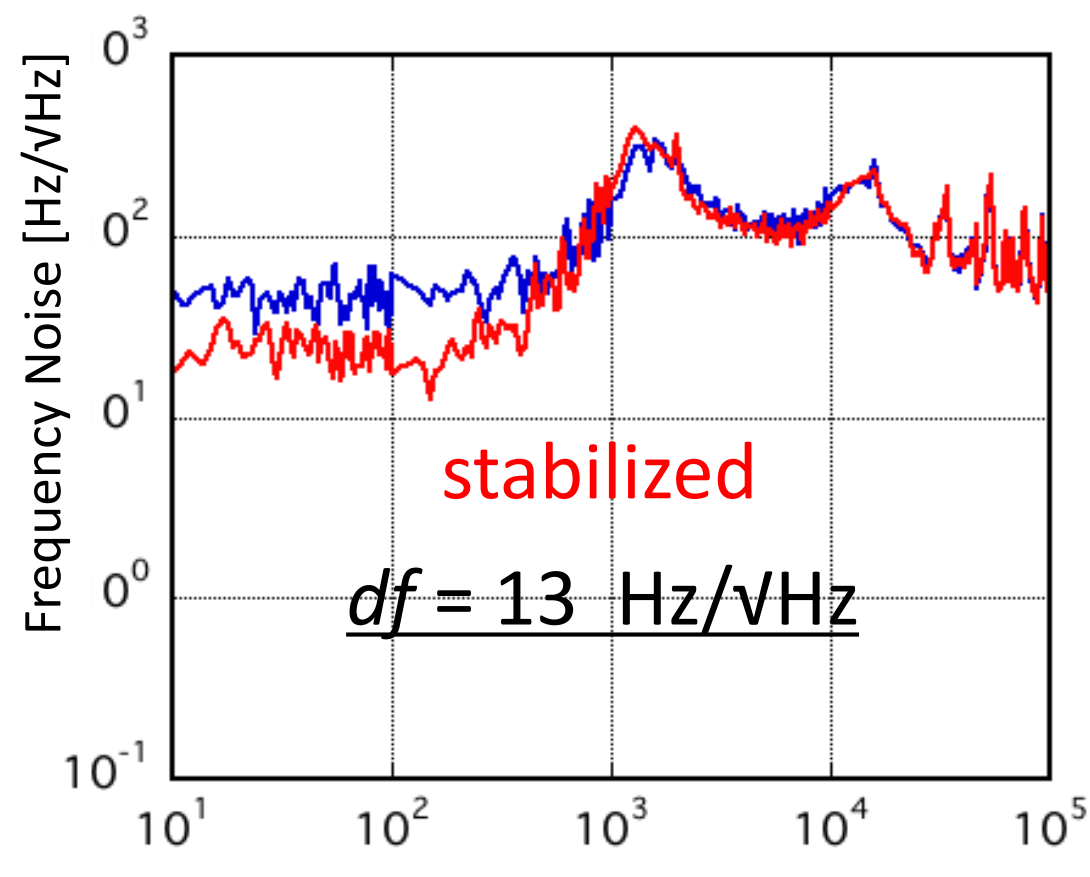

Frequency $[\mathrm{Hz}]$ 


\section{Outline}

- Gravitational wave (GW) \& space GW detector

- Frequency stabilization

- Balanced detection

- Intensity stabilization at $200 \mathrm{kHz}$

- Cascaded amplifier for B-DECIGO

- Summary \& future prospects 


\section{MOFPA for B-DECIGO}

\section{(Master Oscillator Fiber Power Amplifier)}

MOPA

(Master Oscillator Power Amplifier)

Cascaded amplifier

$\rightarrow$ High SNR signal and high efficiency
FA (Fiber Amplifier)

- High efficiency

- High beam quality

- Small \& robustness

- High polarization stability

- Resistance for cosmic_radiation exposure

$$
2^{\text {nd }} \text { stage }
$$

$1^{\text {st }}$ stage

Pre-amplifier
Main-amplifier

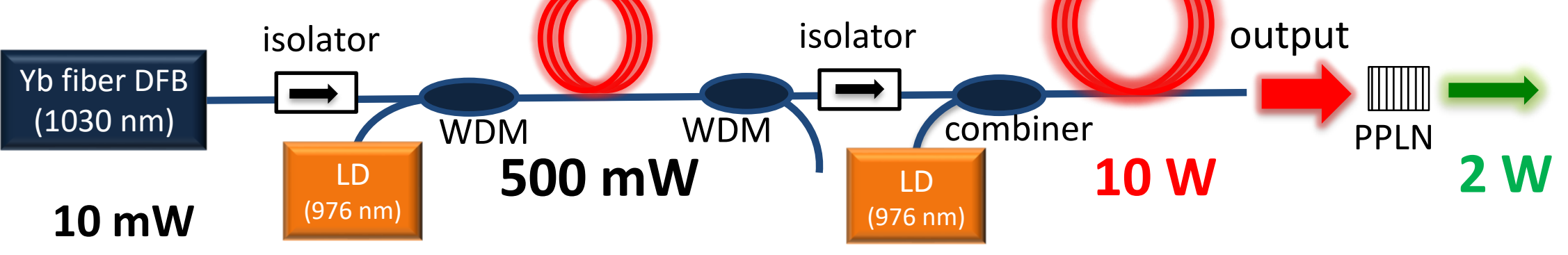

Single clad fiber

Double clad fiber

Core pumping Clad pumping 


\section{Cascaded-amplifier for B-DECIGO}

Clad pumping

Double clad fiber

Core/Clad : $10.0 \mu \mathrm{m} / 125 \pm 2 \mu \mathrm{m}$

Absorption: $7.4 \mathrm{~dB} / \mathrm{m}$

Controlling the stimulated Brillouin scattering (SBS)

- Large core fiber $(10 \mu \mathrm{m})$

- Shorten fiber length $(3.8 \mathrm{~m})$
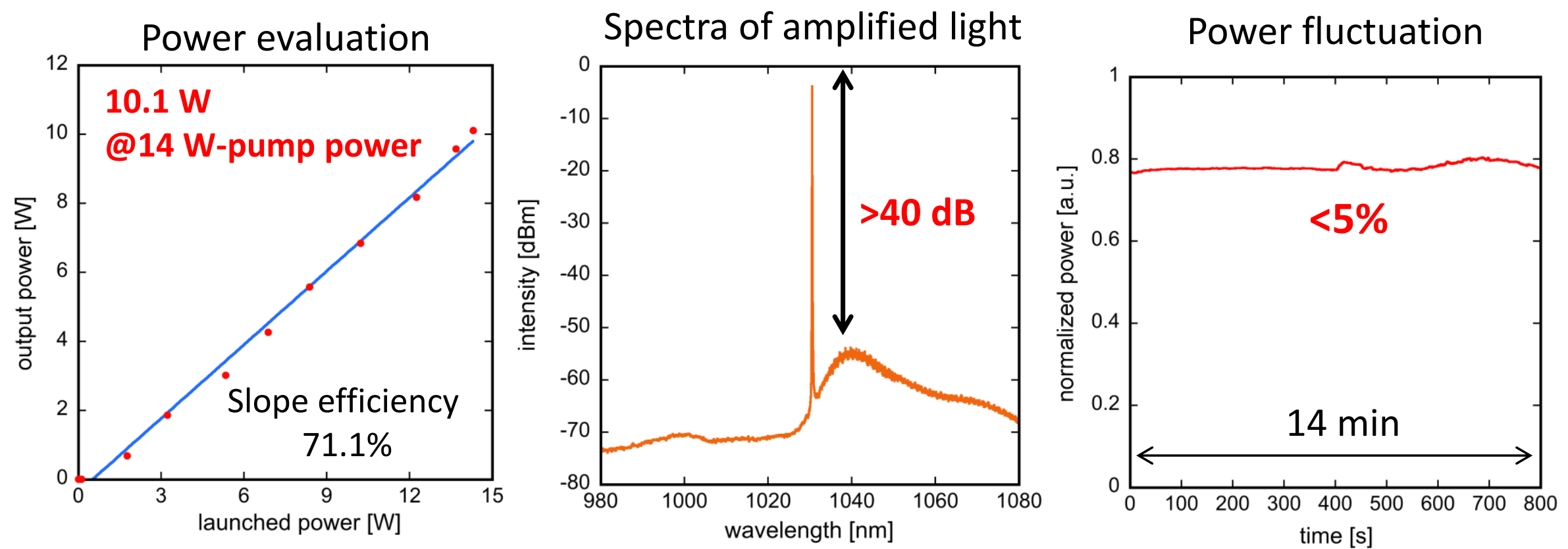

Satisfy the required level of B-DECIGO (1030 nm) 


\section{Beam quality}

Vending the gain fiber to eliminate the higher-order transmission mode

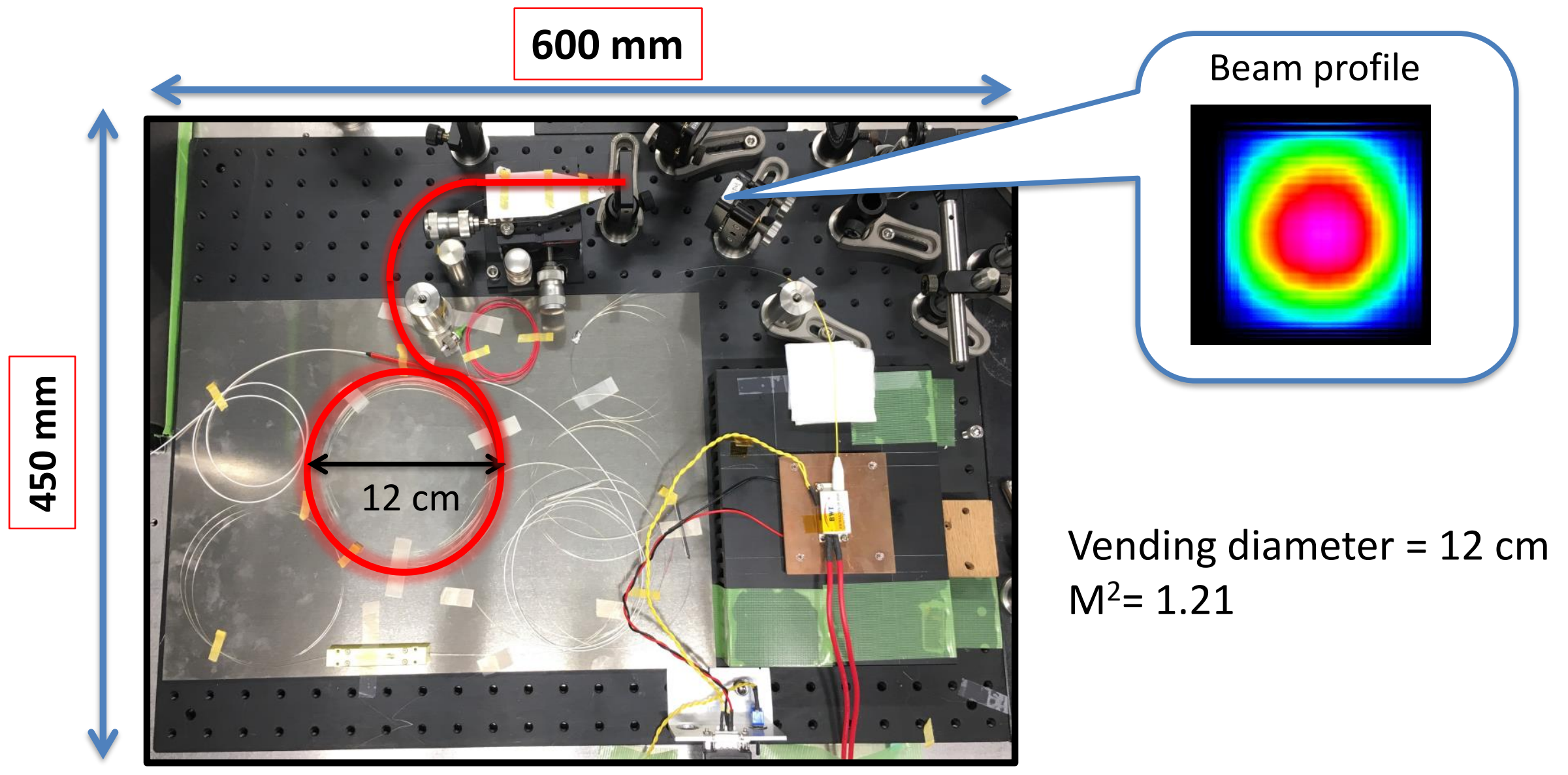




\section{Polarization characteristic}

\section{Polarization extinction ratio}

Fiber DFB $30 \mathrm{~dB}$

Pre amplifier $28.7 \mathrm{~dB}$

Main amplifier $23.7 \mathrm{~dB}$

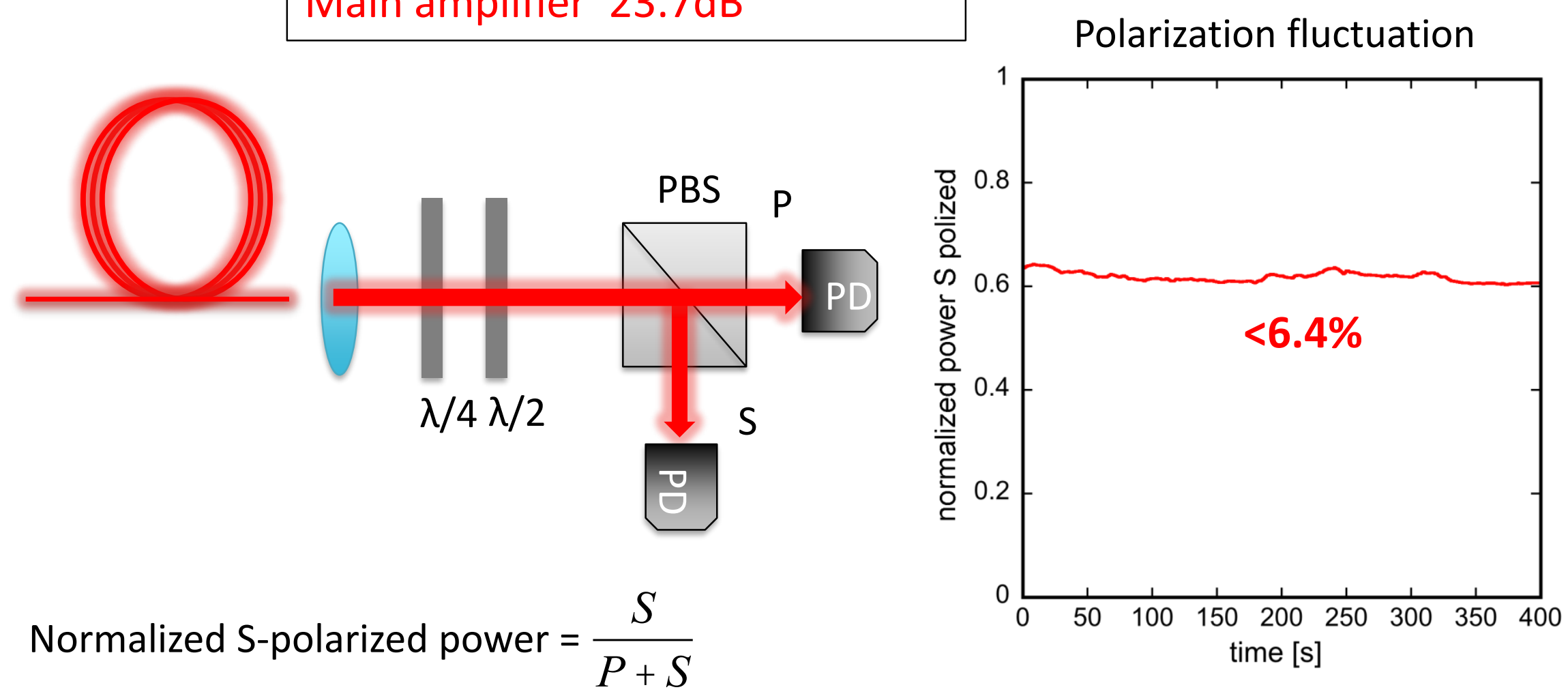




\section{Future prospects}

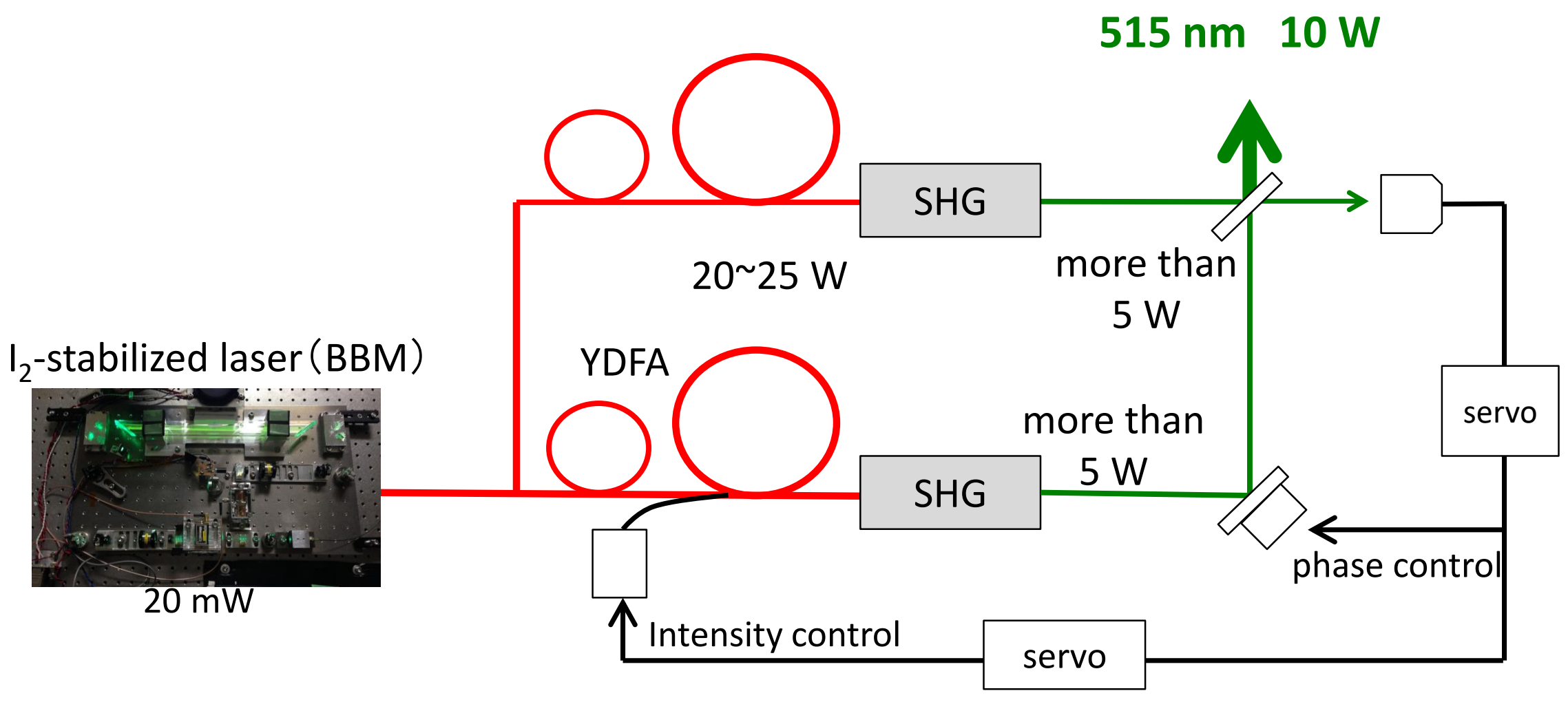

$10 \mathrm{~W}$ green light will be obtained by coherent addition of cascaded amplifiers. 


\section{Outline}

- Gravitational wave (GW) \& space GW detector

- Frequency stabilization

- Balanced detection

- Intensity stabilization at $200 \mathrm{kHz}$

- Cascaded amplifier for B-DECIGO

- Summary \& future prospects 


\section{Summary}

Developments of light source for DECIGO/B-DECIGO Frequency stabilization

Laser frequency was locked to $\mathrm{I}_{2}$-saturated absorption (515 nm)

Frequency noise $\Delta f$ (in-loop) $=1 \mathrm{~Hz} / \mathrm{VHz}$ (BBM1,BBM2) ... ()

Frequency noise $\Delta$ f(out-of-loop) $=30 \mathrm{~Hz} / \mathrm{VHz} \ldots \triangle$

Allan deviation of beat signal of 2 BBMs : $\Delta f / f=10^{-13}(1 \mathrm{~s}), \Delta f / f=10^{-14}(1000 \mathrm{~s}) \ldots \Delta$

Intensity stabilization

(1)around $1 \mathrm{~Hz}$ (observation band, low power)

$\mathrm{RIN}: \Delta \mathrm{I} / \mathrm{I}=5 \times 10^{-8} / \mathrm{VHz}$ (fiber components experiments) ...

(2)at modulation band $(200 \mathrm{kHz}) \ldots$

High power

$10 \mathrm{~W}$ of $1030 \mathrm{~nm}$-light is obtained... $\bigcirc$ 


\section{Acknowledgements}

This research is supported by

the Japan Aerospace Exploration Agency (JAXA), and the Japan Society for the Promotion of Science (JSPS), Grand-in-Aid for Scientific Research

\section{Thank you for your attention!}




\section{Future prospects (2)}

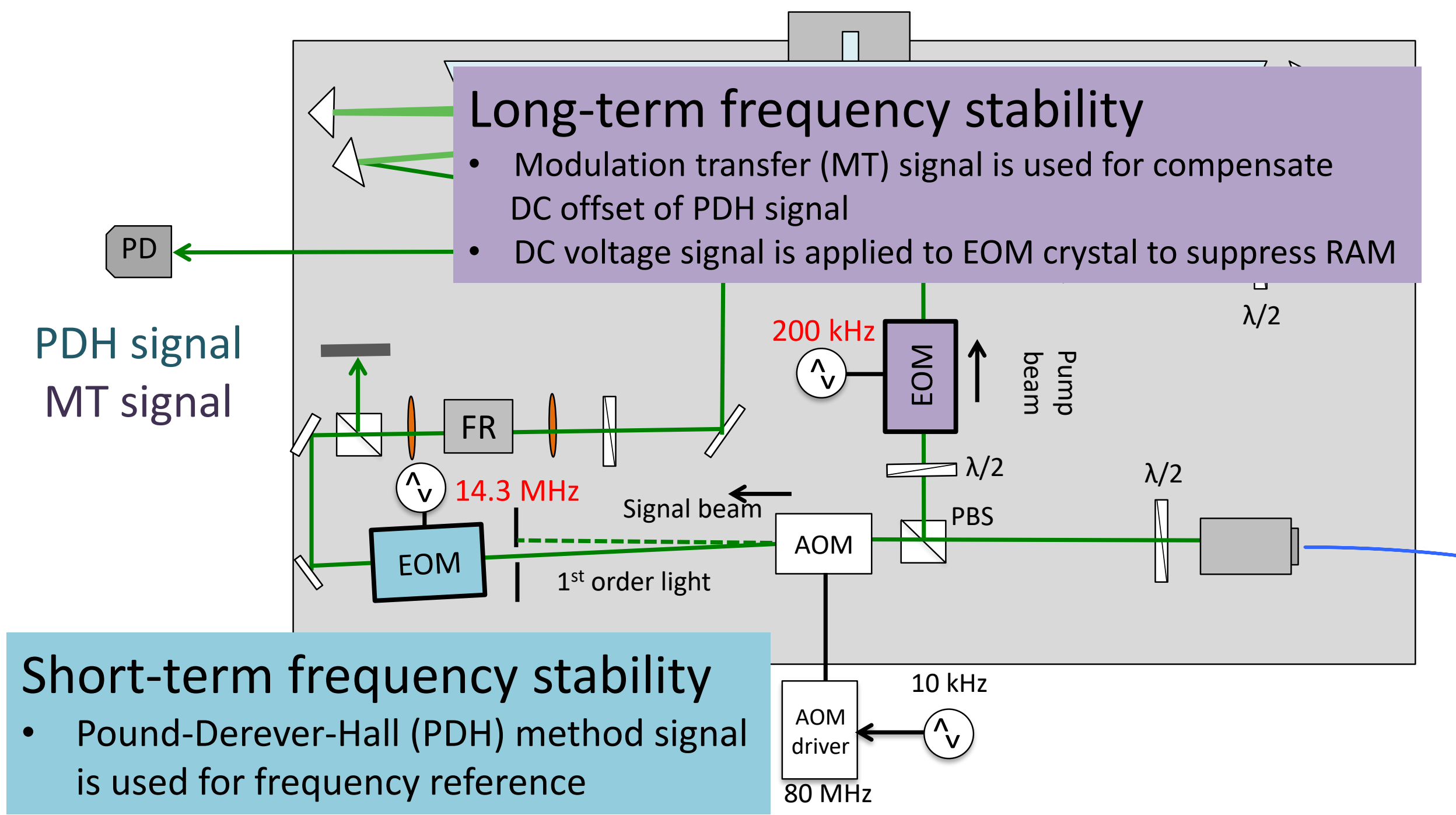

ICSO2018 9/10/2018@Crete 


\section{Pre-amplifier}

Max power = 420 mW @700 mW-pump power $)$ Forward-pumping and core pumping Single clad fiber $\mathrm{SNR}=50 \mathrm{~dB}$

Core/clad:6.0 $\mu \mathrm{m} / 125 \pm 2 \mu \mathrm{m}$

Fiber length $1.5 \mathrm{~m}$
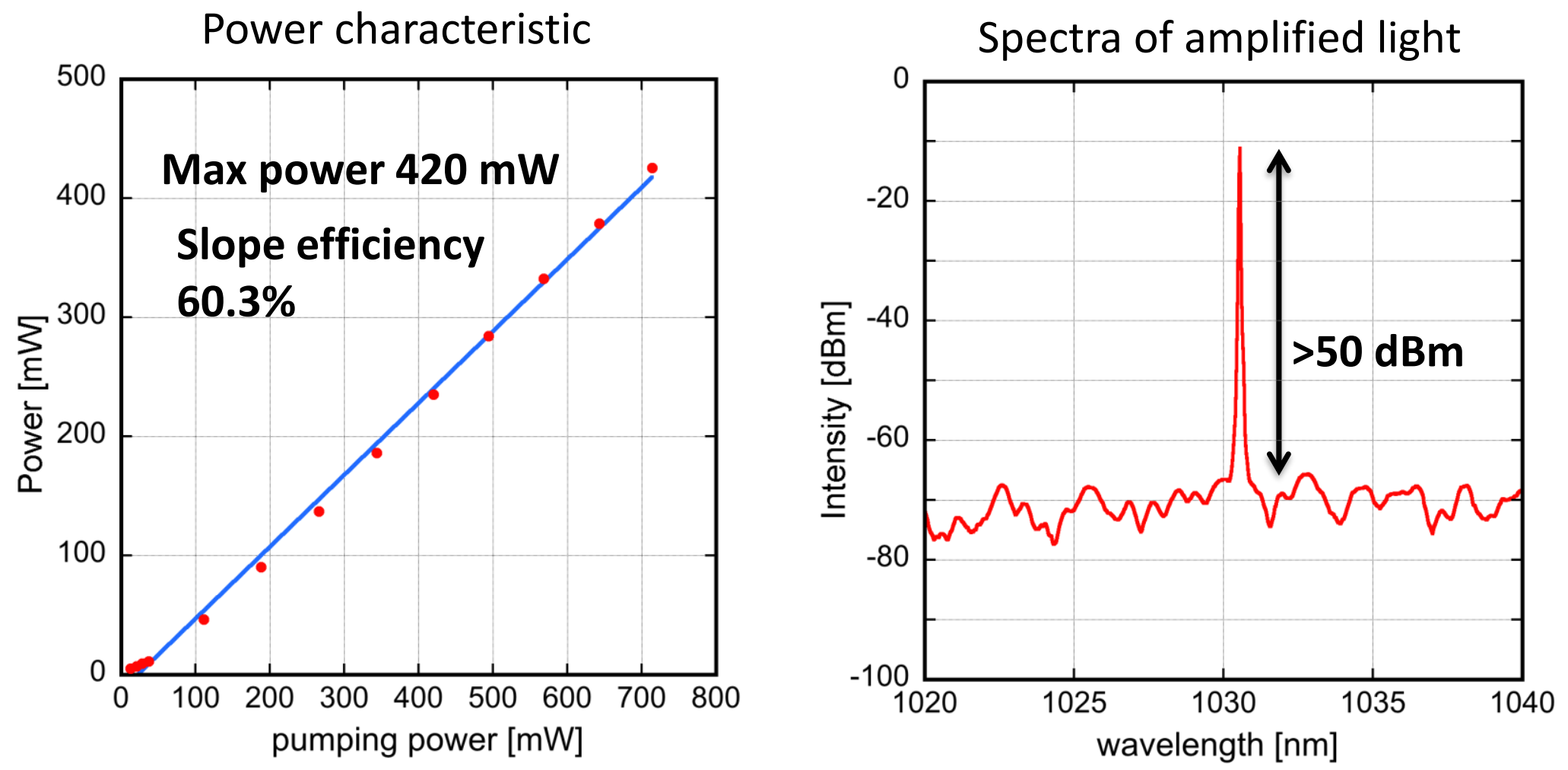


\section{Intensity stabilization @ 1Hz}

Stabilization at $1 \mathrm{~Hz}$ is realized by controlling the power of YDFA

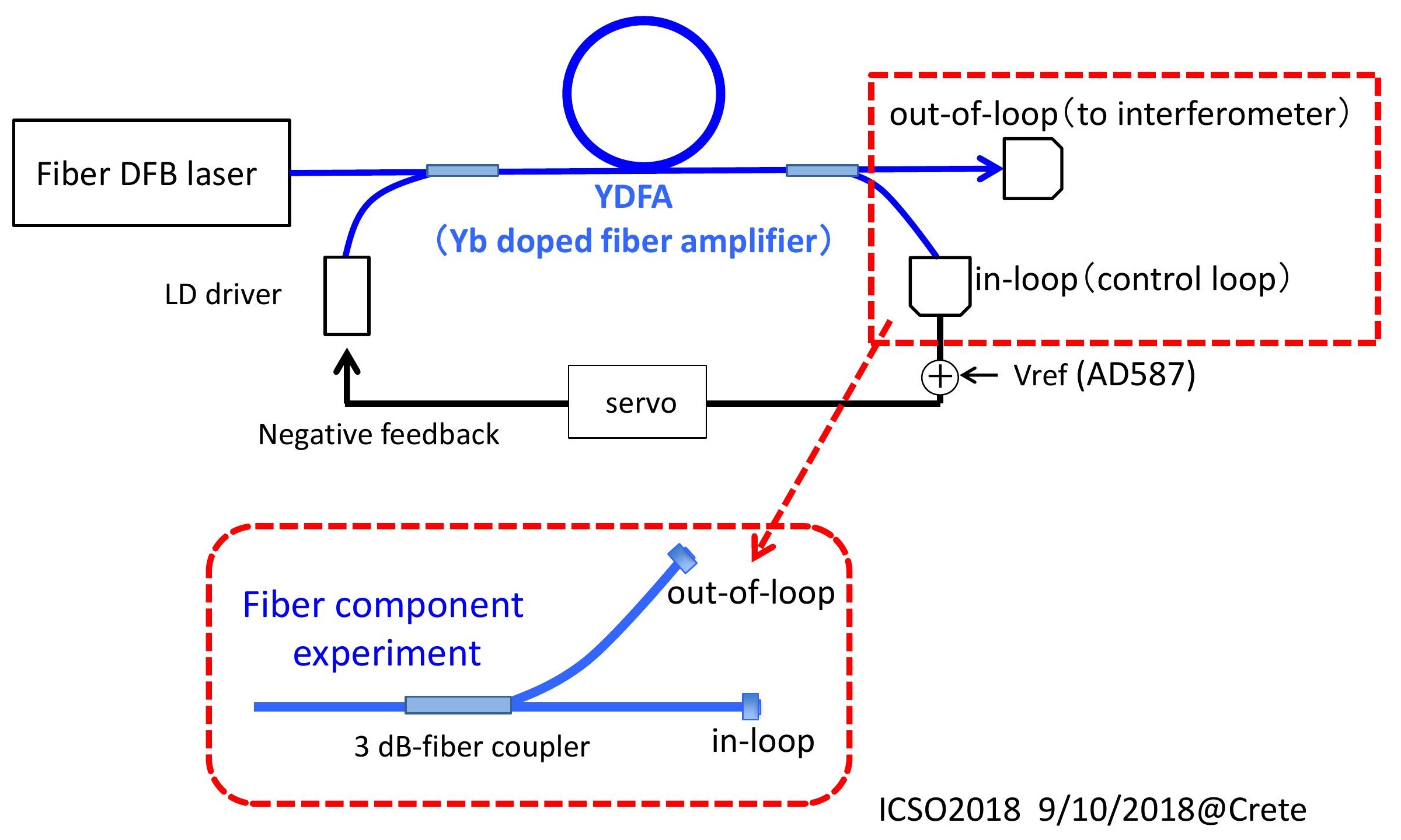




\section{Intensity stabilization @ 1 Hz}

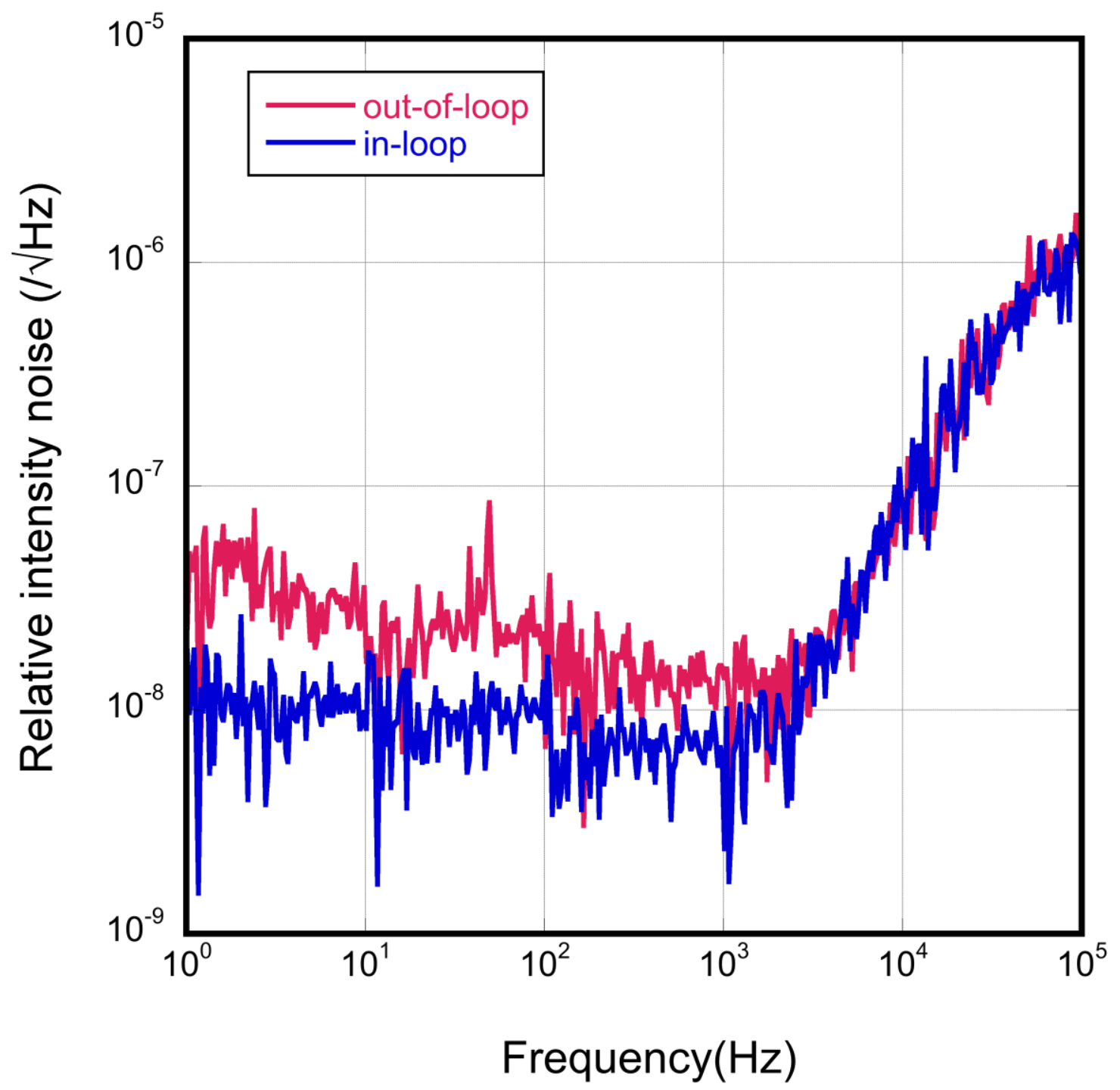




\section{Balanced detection}

The circuit diagram of the automatic balanced photo detector

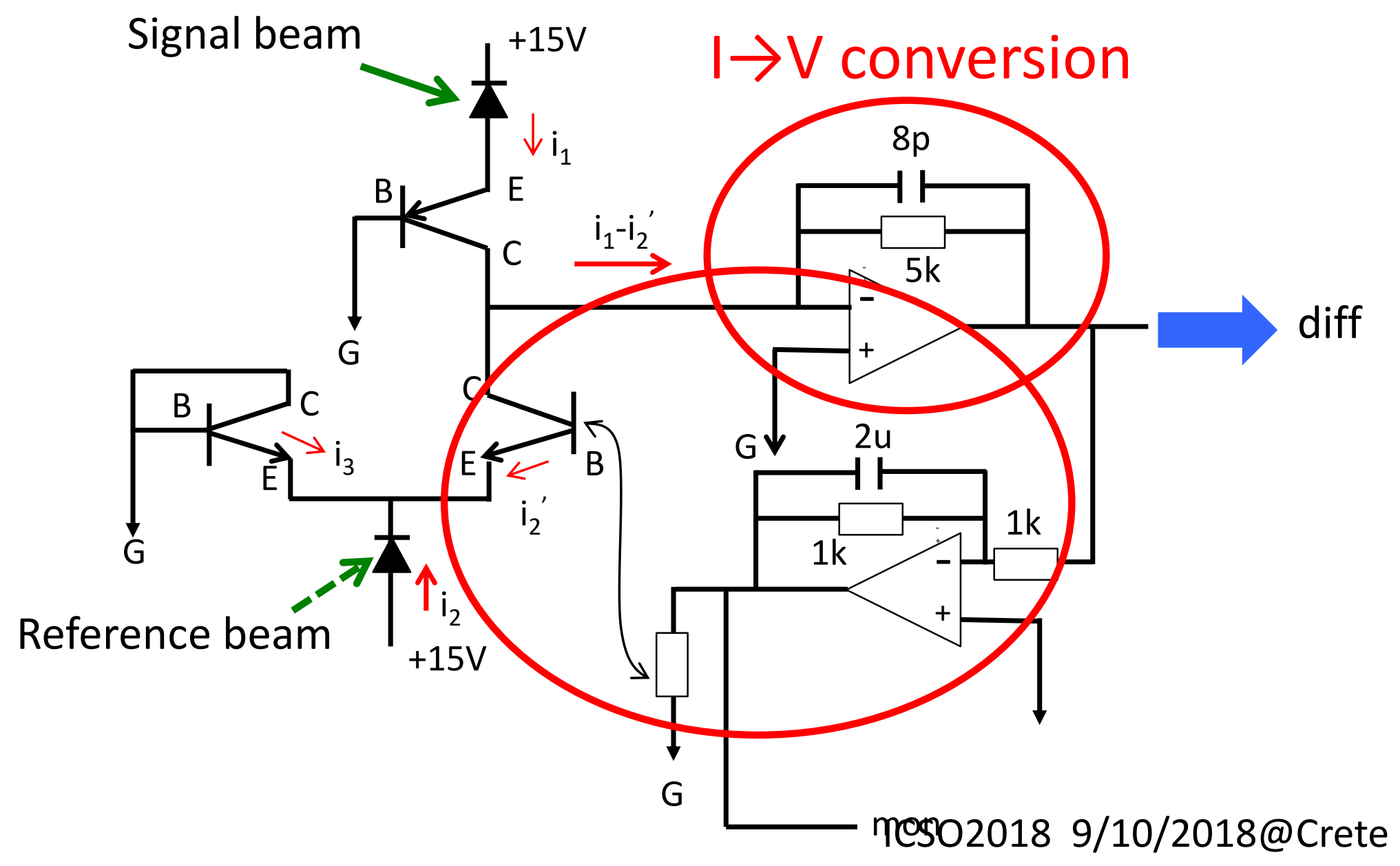




\section{Japan GW detection projects}

\section{DECIGO/B-DECIGO}

Space GW detector

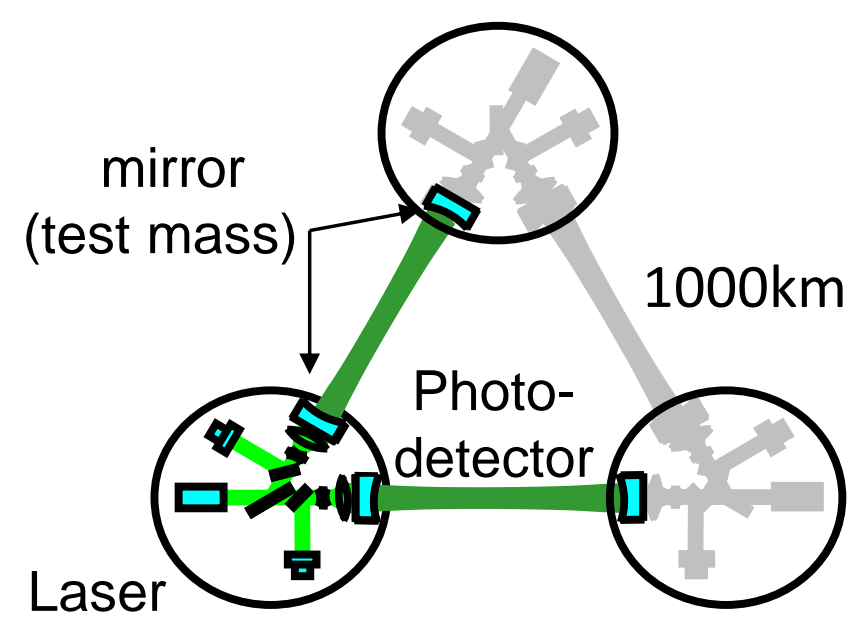

- Strain sensitivity : $\Delta L / L=10^{-23 / V H z}$

- Observation band :0.1 10 Hz

- Planed to be launched in 2035

- Target: IMBH binary, NS binary,

BGGW from early universe 


\section{B-DECIGO}

$\mathrm{I}_{2}$-stabilized laser (BBM)

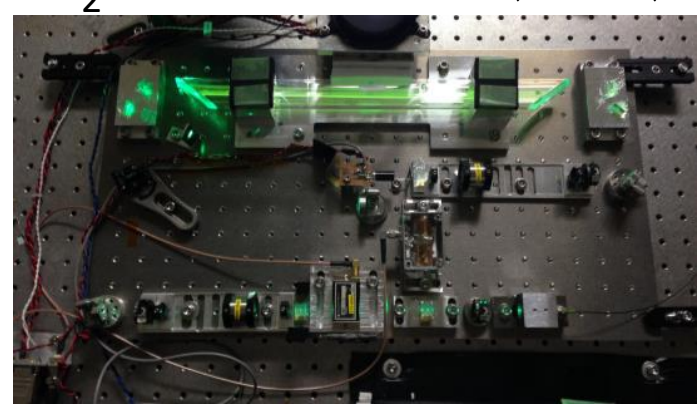

cascaded amplifier rre-Amp + rower Amp

$+$

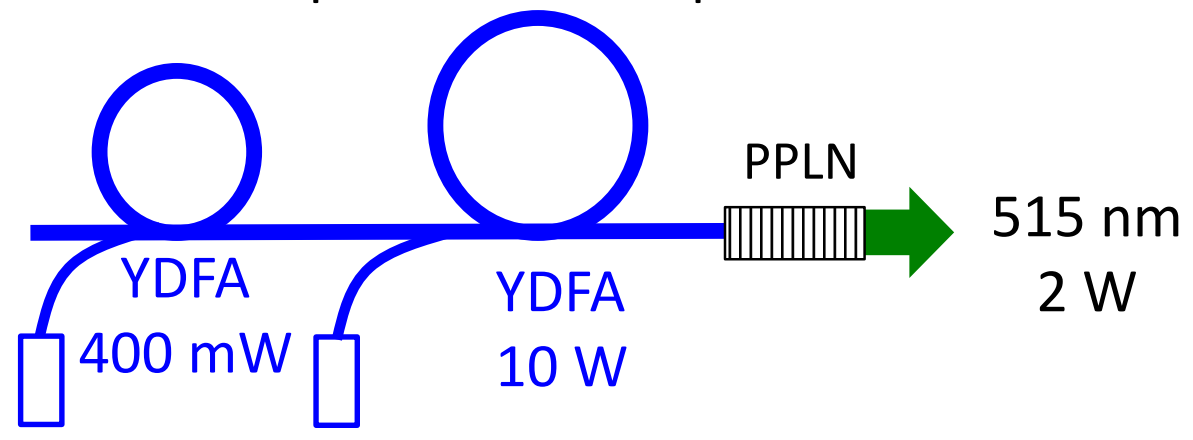




\section{Intensity-stabilization_@1Hz}

relative intensity noise spectrum

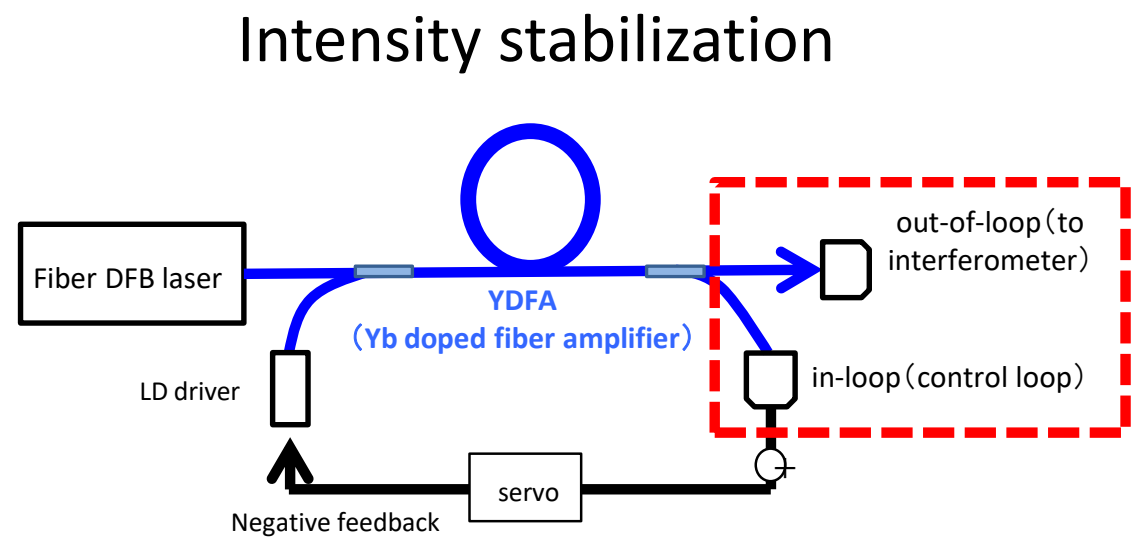

Differential mechanical vibration of fibers
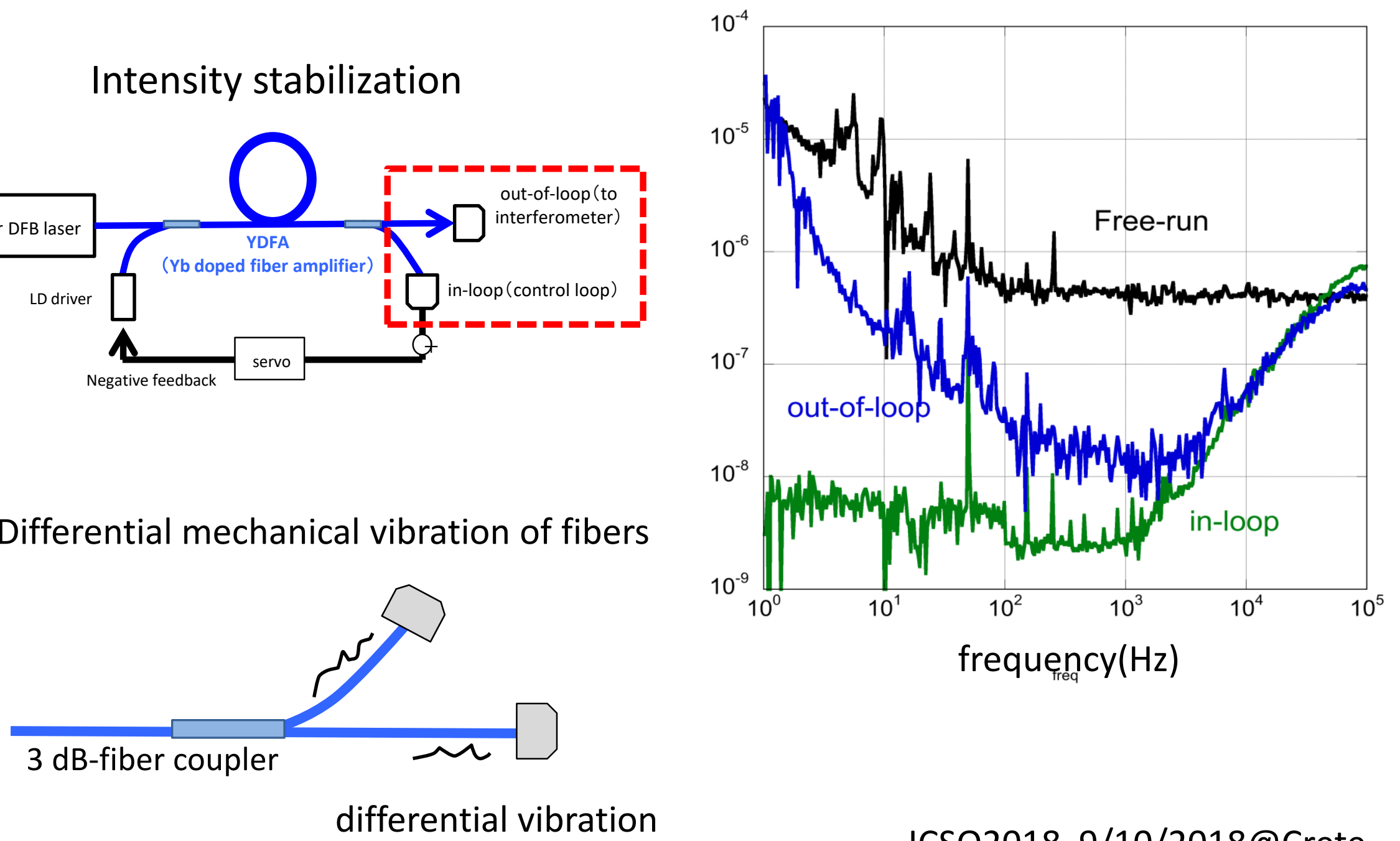


\section{変調周波数での強度安定化-feed forward(2)-}

$\diamond$ 強度安定化結果(feed forward)
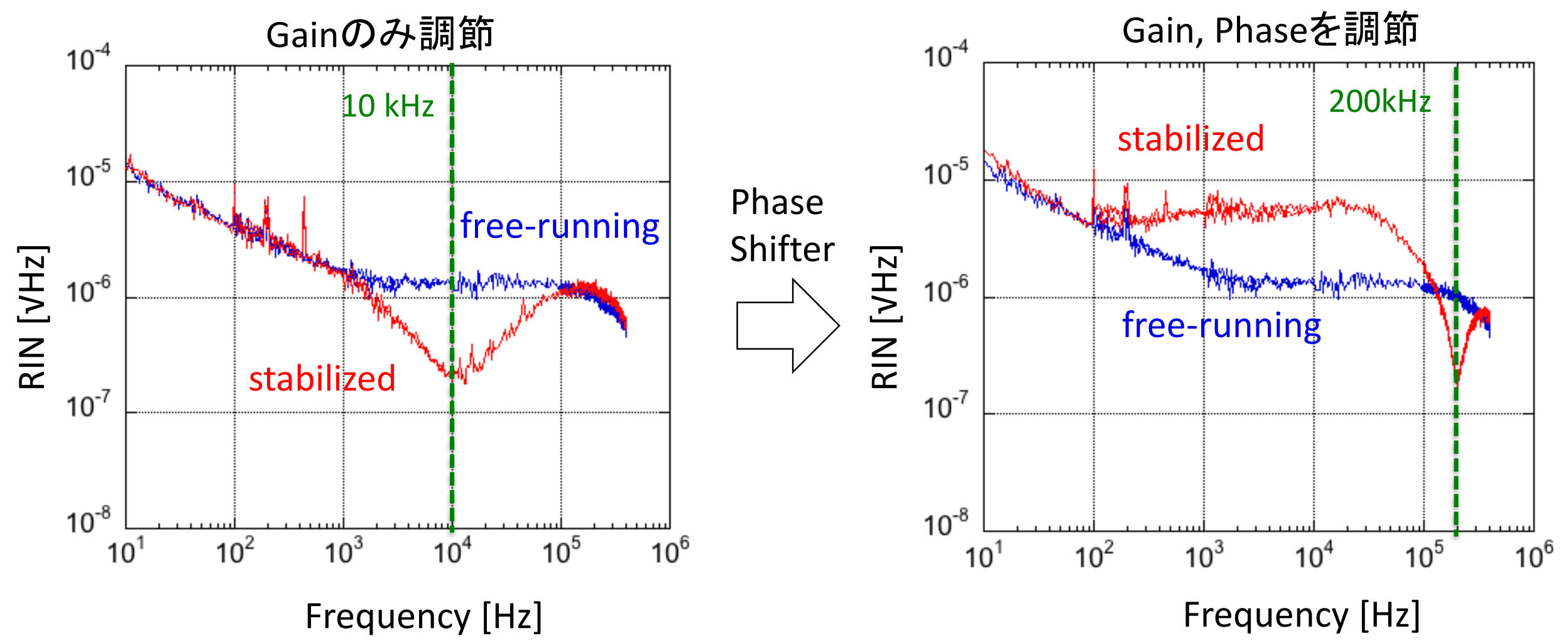

$1.7 \times 10^{-7} / \mathrm{VHz} @ 200$ kHzの安定度を達成 今後はこの系で周波数安定化を行う 


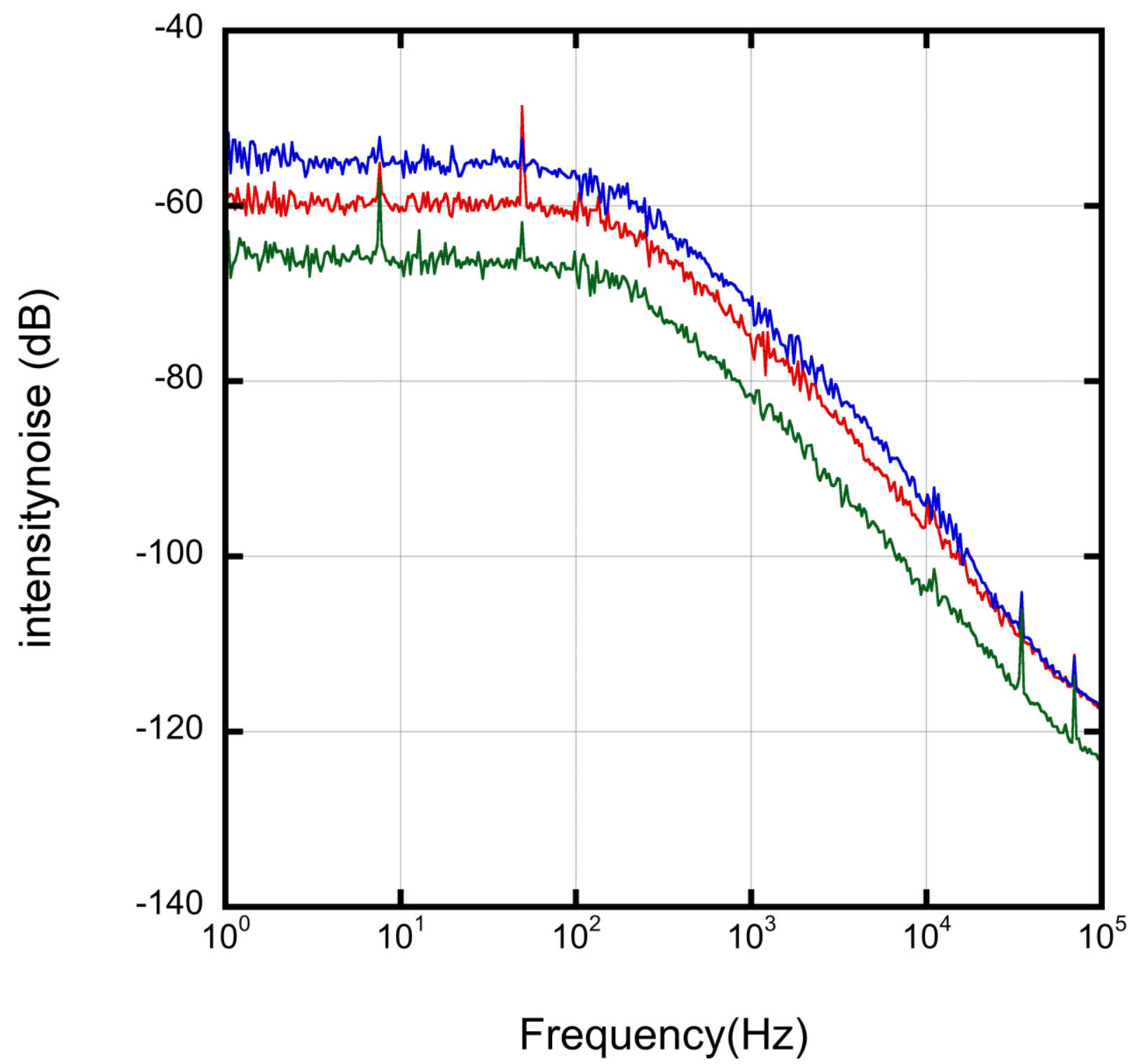

ICSO2018 9/10/2018@Crete 


\section{Intensity stabilization@200kHz}

Schematic of feed forward method

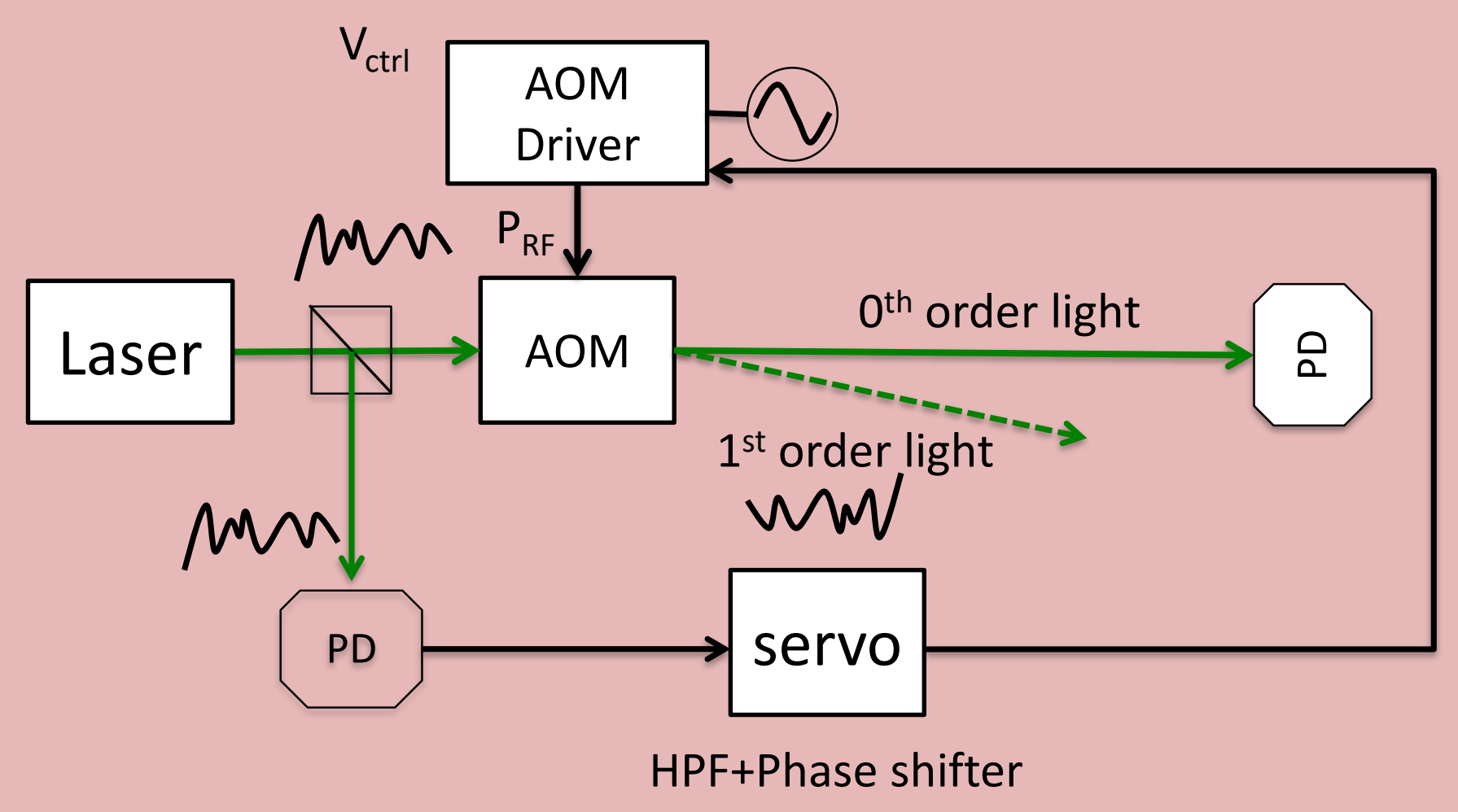




\section{KAGRA}

\section{Ground-based GW detector}

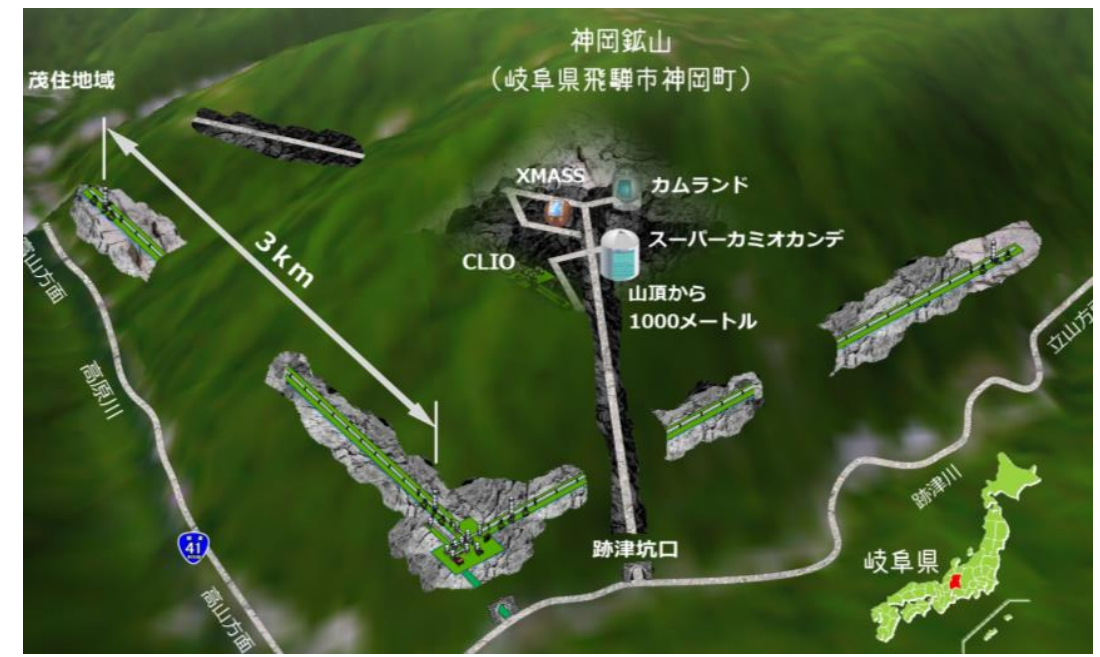

- Kamioka mine in Gifu, Japan

- Initial interferometer (i-KAGRA) was completed in this spring

- Now under improvement for observation run 


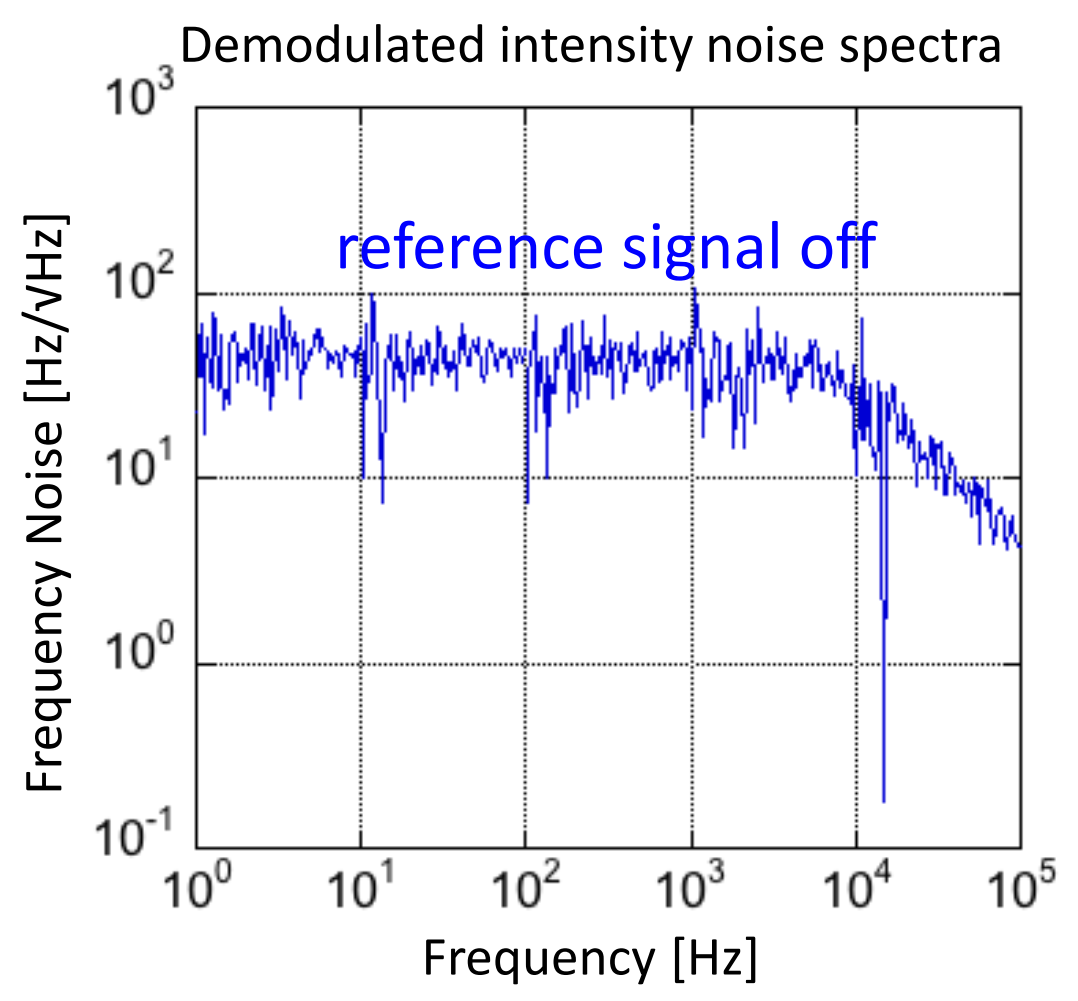

ICSO20189/10/2018@Crete 


\section{差動受光(2)}

々 周波数制御時の差動受光によるパフォーマンス

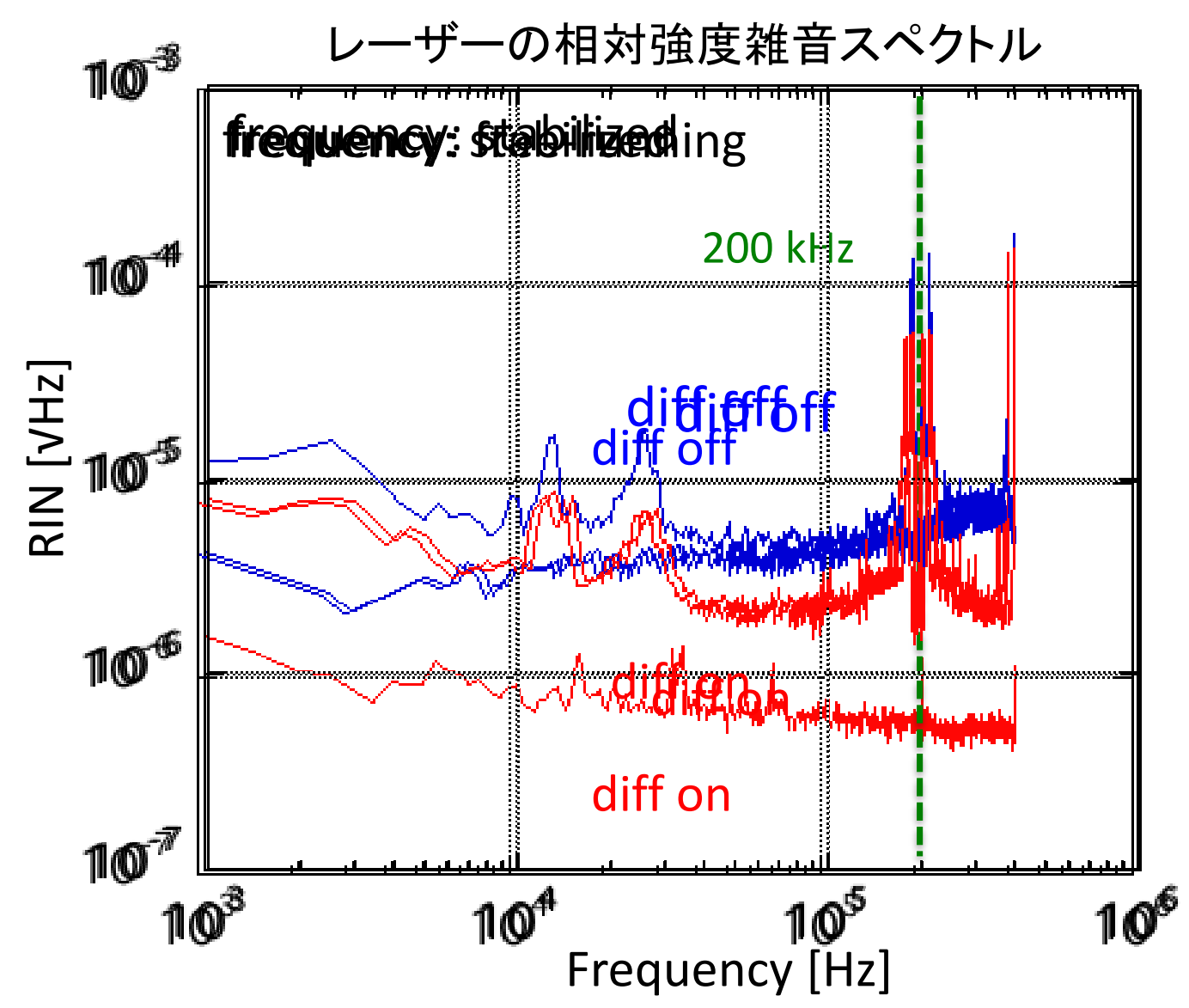

無制御時より雑音抑圧利得は小さくなるものの $6 \mathrm{~dB}$ 程度の雑音を抑圧 